\title{
A spatial model for conflict incorporating within- and between-actor effects
}

\author{
Diána Knipl ${ }^{\mathrm{a}, \mathrm{b}}$, Toby Davies ${ }^{\mathrm{c}, *}$, Peter Baudains ${ }^{\mathrm{c}}$ \\ ${ }^{a}$ Department of Mathematics, University College London, Gower Street, London, WC1E \\ 6BT, United Kingdom \\ ${ }^{b}$ MTA-SZTE Analysis 8 Stochastic Research Group, University of Szeged, Aradi \\ vértanúk tere 1, 6720 Szeged, Hungary \\ ${ }^{c}$ Department of Security $\&$ Crime Science, University College London, Gower Street, \\ London, WC1E 6BT, United Kingdom
}

\begin{abstract}
The application of ecological models to human conflict scenarios has given rise to a number of models which describe antagonistic relationships between adversaries. Recent work demonstrates that the spatial disaggregation of such models is not only well-motivated but also gives rise to interesting dynamic behaviour, particularly with respect to the spatial distribution of resources. One feature which is largely absent from previous models, however, is the ability of an adversary to coordinate activity across its various locations. Most immediately, this corresponds to the notion of 'support' - the reallocation of resources from one site to another according to need - which plays an important role in real-world conflict. In this paper, we generalise a spatiallydisaggregated form of the classic Richardson model of conflict escalation by adding a cross-location interaction term for the within-adversary dynamics at each location. We explore the model analytically, giving conditions for the stability of the balanced equilibrium state. We then also carry out a number of numerical simulations which correspond to stylised real-world conflict scenarios. Potential further applications of the model, and its implications for policy, are then discussed.
\end{abstract}

Keywords: conflict, reaction-diffusion, within-adversary action, stability analysis

\footnotetext{
${ }^{*}$ Corresponding author.
} 


\section{Introduction}

Over the last century, human conflict has become an increasingly popular topic for mathematical modelling. Both outbreaks of conflict themselves and the interactions that lead to them display significant dynamics which, in many cases, invite modelling by analogy with other systems, particularly those within ecology. The ability to capture these dynamics using mathematical models has significant potential value from the perspectives of research and policy: as well as offering insight into the behaviours involved, such models can be used to investigate strategies for conflict resolution and avoidance.

The majority of conflict modelling to date has been based on the adaptation of ecological models which mimic the competition between species, since both feature growth and decay elements, coupled to reflect the interactions between distinct entities. This approach is exemplified by the two landmark models which inspired much of the subsequent interest in the field: Lanchester's [1] model of attrition in warfare and Richardson's [2] model of conflict escalation. In both of these cases, the evolution of a resource (respectively, manpower and military expenditure) is described using equations which incorporate both internal and external influences. This general approach closely related to the Lotka-Volterra model of predator-prey interaction has since been refined and adapted to apply to a number of different types of conflict $[3,4,5,6,7,8,9,10,11,12]$.

One feature which is absent from the majority of conflict models is the influence of space: most approaches are based on an assumption of "wellmixedness', and describe the dynamics purely in terms of total resource levels. This assumption, however, neglects the important role played by spatial configuration in conflict scenarios. In general, the ability of one entity to exert either violence or threat upon another is inversely proportional to the distance between them. This observation, coupled with the fact that entities participating in conflict are often spread over a number of locations (e.g. territories or bases), suggests that conflict is highly dependent on nontrivial spatial interactions, and this undermines approaches which consider only total resource levels.

In order to address these issues, a spatially-disaggregated variation of the classic Richardson model has recently been introduced [13]. In this model, each adversary has resources situated at multiple locations on a 2-dimensional manifold; that is, its total resources are spread over a number of sites. The central idea of the model is to describe how the total effect emanating from 
any location is allocated across the locations of the adversary; that is, how much threat is felt at location $j$ as a result of an opponent's military capability at $i$. This threat level is estimated using an entropy-maximising approach, and the influence acts as the coupling term for a system of Richardson equations describing the behaviour at each location. Analysis of the model reveals a bifurcation as the parameter representing aggression is increased, in which the spatial distribution of resources at equilibrium becomes highly concentrated on a small number of locations.

The spatial Richardson model is still, however, unrealistic in some respects. In particular, it fails to account for an individual adversary's ability to coordinate resources across its locations: while external interactions are coupled, internal effects act independently at each location. In reality, however, locations are not autonomous in this respect. An adversary's activities at each location are typically coordinated by a central authority which, in particular, has the ability to reallocate resources from one site to another. This represents the provision of 'support': if an adversary is experiencing threat at one of its locations, it can bolster its presence by moving resources from locations where it is less exposed. In mathematical terms, this requires the introduction of coupling in the within-adversary growth effects at each location.

In this paper, we propose a new spatial model for the evolution of conflict, incorporating both inter-adversary hostility and within-adversary support. The inclusion of support is achieved via the addition of within-adversary coupling to the internal reaction term, acting alongside the inter-adversary antagonistic effects of the original Richardson model. The form of this term is based on entropy-maximisation principles, and the resulting model represents a general framework for the modelling of hostile interactions. After presenting the model, we analytically examine a number of low-dimensional, simplified forms. We establish a number of stability conditions in these settings, which reveal in particular the effect of the support term on the longterm dynamics of the system. Finally, we examine the behaviour of the model numerically: we demonstrate our basic analytical findings, and additionally examine the behaviour of the model in a number of higher-order configurations selected to correspond to real-world conflict scenarios. 


\section{Model formulation}

In 1960, Richardson proposed the following model to describe the levels of military expenditure of two nations (denoted by $p$ and $q$ ):

$$
\begin{aligned}
& \dot{p}=-\sigma_{1} p+\rho_{1} q+\epsilon_{1}, \\
& \dot{q}=\rho_{2} p-\sigma_{2} q+\epsilon_{2},
\end{aligned}
$$

where $\sigma_{1}, \sigma_{2}, \rho_{1}, \rho_{2}, \epsilon_{1}$ and $\epsilon_{2}$ are parameters, and the overdot is the usual notation for the time derivative. Each equation incorporates three effects, relating to the acquisition and disposal of military resources. The internal response term (parameterised by $\sigma_{1}$ and $\sigma_{2}$ ) reflects the influence of existing spending levels on the acquisition rate. Both $\sigma_{1}$ and $\sigma_{2}$ are typically taken to be positive, reflecting some notion of impedance associated with the accumulation of arms: this may result either from an underlying tendency towards non-aggression, or simply reflect depreciation in the value of existing arms. The action-reaction term (determined by $\rho_{1}$ and $\rho_{2}$ ) encodes the interaction between the nations; that is, the response of each to the acquisitions of the other. In most scenarios, $\rho_{1}$ and $\rho_{2}$ are also taken to be positive, reflecting antagonism between the adversaries: each responds to the other's expenditure by increasing its own spending. Finally, the terms $\epsilon_{1}$ and $\epsilon_{2}$ represent the effect of external grievances, such as the threat posed by exogenous actors.

The dynamics of this basic system were studied by Richardson, and a number of properties were established. Most immediately, under the assumption $\rho_{1} \rho_{2} \neq \sigma_{1} \sigma_{2}$, the system can be shown to have a unique equilibrium $(\bar{p}, \bar{q})$, where

$$
\bar{p}=\frac{\sigma_{2} \epsilon_{1}+\rho_{1} \epsilon_{2}}{\sigma_{1} \sigma_{2}-\rho_{1} \rho_{2}}, \quad \bar{q}=\frac{\rho_{2} \epsilon_{1}+\sigma_{1} \epsilon_{2}}{\sigma_{1} \sigma_{2}-\rho_{1} \rho_{2}},
$$

and all solutions converge to $(\bar{p}, \bar{q})$ provided that $\rho_{1} \rho_{2}<\sigma_{1} \sigma_{2}$ holds. In terms of real-world behaviour, this implies that perpetually escalating 'arms races' will only occur if the tendency to aggression is sufficiently large, with the two nations otherwise settling towards a deadlock of mutually-tolerable threat.

Taking this model as a basis, we formulate a new model of arms expenditure between two actors which takes into account the effect of space in moderating conflict interactions. We do this by disaggregating the model in a way which reflects the distribution of each actor's resources in space, and incorporates spatial interaction effects in the behaviour of each adversary.

We consider a situation involving two adversaries, $P$ and $Q$, each of which possesses resources at a number of discrete locations. The spatial setting for 


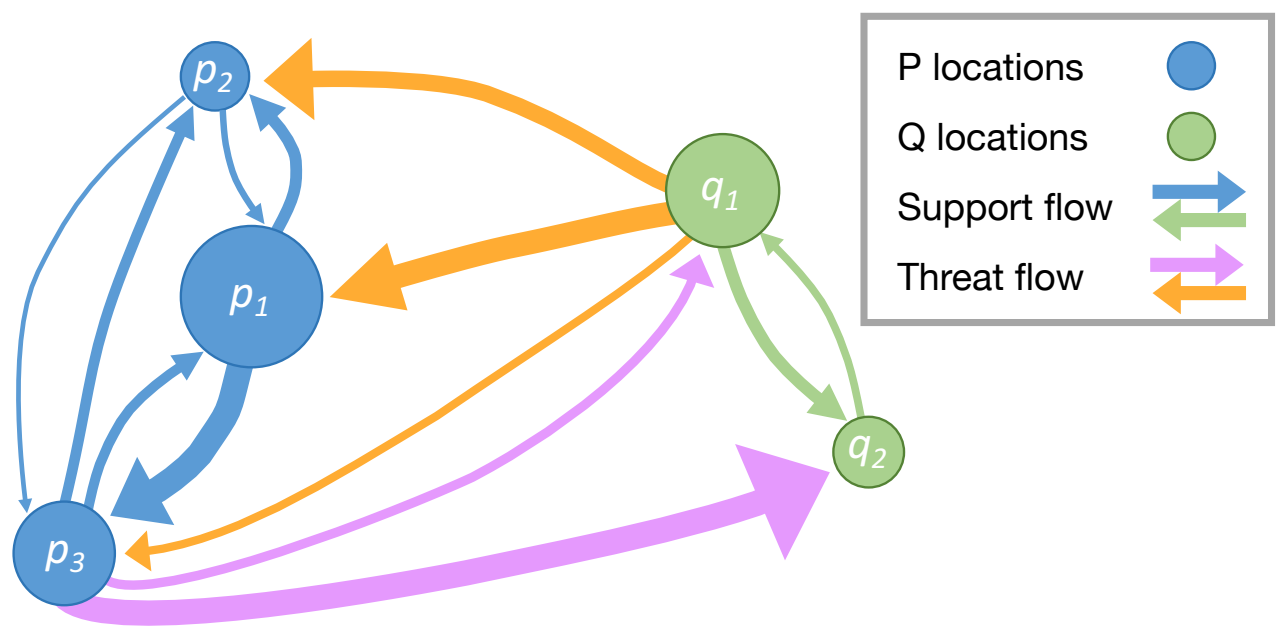

Figure 1: Schematic diagram illustrating the structure of flows in a hypothetical configuration of the model with $N=3$ and $M=2$. The size of each location indicates its level of expenditure, and the widths of the arrows represent the strength of the within- and between-adversary effects. For clarity, the threat flows from only two locations are shown.

the model is a two-dimensional manifold, $\mathcal{M}$, which represents the domain in which the conflict takes place. Within this, each adversary controls a number of sites: $P$ is taken to be located at $N$ spatially discrete points $x_{1}$, $\ldots, x_{N} \in \mathcal{M}$, and $Q$ is taken to be located at $M$ spatially discrete points $y_{1}$, $\ldots, y_{M} \in \mathcal{M}$.

We use the term $p_{j}$ to denote the level of $P$ 's hostility at location $x_{j}$ for $j=1, \ldots, N$, and $q_{l}$ to denote the level of $Q$ 's hostility at location $y_{l}$ for $l=1, \ldots, M$. For each $j \in\{1, \ldots, N\}$ and for each $l \in\{1, \ldots, M\}$, the 
dynamics of $p_{j}$ and $q_{l}$ are described by the following equations:

$$
\begin{aligned}
\dot{p}_{j}= & -\sigma_{1} p_{j}-\phi_{1} p_{j}+\phi_{1} \sum_{i=1}^{N} p_{i} \frac{p_{j}^{a} e^{-\alpha \cdot d\left(x_{j}, x_{i}\right)}}{\sum_{j^{\prime}=1}^{N} p_{j^{\prime}}^{a} e^{-\alpha \cdot d\left(x_{j^{\prime}}, x_{i}\right)}} \\
& +\rho_{1} \sum_{k=1}^{M} q_{k} \frac{p_{j}^{b} e^{-\beta \cdot d\left(x_{j}, y_{k}\right)}}{\sum_{i=1}^{N} p_{i}^{b} e^{-\beta \cdot d\left(x_{i}, y_{k}\right)}}+\frac{\epsilon_{1}}{N} \\
\dot{q}_{l}= & -\sigma_{2} q_{l}-\phi_{2} q_{l}+\phi_{2} \sum_{k=1}^{M} q_{k} \frac{q_{l}^{a} e^{-\alpha \cdot d\left(y_{l}, y_{k}\right)}}{\sum_{l^{\prime}=1}^{M} q_{l^{\prime}}^{a} e^{-\alpha \cdot d\left(y_{l^{\prime}}, y_{k}\right)}} \\
& +\rho_{2} \sum_{i=1}^{N} p_{i} \frac{q_{l}^{b} e^{-\beta \cdot d\left(x_{i}, y_{l}\right)}}{\sum_{k=1}^{M} q_{k}^{b} e^{-\beta \cdot d\left(x_{i}, y_{k}\right)}}+\frac{\epsilon_{2}}{M}
\end{aligned}
$$

where $\sigma_{1}, \sigma_{2}, \rho_{1}, \rho_{2}, \epsilon_{1}$ and $\epsilon_{2}$ are the same parameters as in the non-spatial model (1), and $a, b, \alpha, \beta, \phi_{1}$ and $\phi_{2}$ are new parameters. We use $d$ to denote a metric, defined on $\mathcal{M} \times \mathcal{M}$, for the distance between locations; since the locations of each adversary are distinct, it is reasonable to assume that $d\left(x_{j}, x_{j}\right)=0$ for all $j$, and that $d\left(x_{j}, x_{i}\right)>0$ if $j \neq i$. Similarly, $d\left(y_{l}, y_{l}\right)=0$ for all $l$ and $d\left(y_{l}, y_{k}\right)>0$ if $k \neq l$.

As in the original Richardson formulation, the model includes a term describing the reaction of each adversary to military spending by the other. Unlike in the aggregated form, however, this is modelled at each location: each site reacts independently to the threat it experiences. In order to do this, it is necessary to estimate how the total threat emanating from resources deployed at any given location is divided across the locations of the adversary. This is done using an entropy-maximising method, described in Ref. [13], which estimates the most likely flow of threat between locations. This encapsulates two key principles: the flow of threat towards larger sites (parameterised by $b$ ), and an impedance effect proportional to the distance between locations (parameterised by $\beta$ ). These appear in the model as action-reaction terms, with strength parameterised by $\rho_{1}$ and $\rho_{2}$ : again, these are assumed to be positive, corresponding to antagonistic behaviour.

The model also includes a term which represents within-adversary interaction across locations. This is not present in earlier models, but is included here to reflect the ability of each adversary to coordinate activity across the locations at which its resources are present. Most immediately, this behaviour corresponds to the notion of 'support': the reallocation of resources from one 
site to another according to strategic need. In the real-world, this would typically be achieved through the transport of military personnel or hardware, both of which have some associated cost. We model this using an additional interaction term, derived according to similar principles to those used for the antagonistic behaviour. Again, the key issue is how the resources from one site are to be reallocated and distributed over the same adversary's other locations. This is done according to entropy-maximising principles, with a function incorporating: (i) the existing level of resource at the destination (parameterised by $a$ ), and (ii) the distance between source and destination (parameterised by $\alpha$ ). In particular, if $\phi_{1}$ is the diffusion rate for adversary $P$, then the level of resource allocated to $p_{j}$ from $p_{i}$ is given by

$$
\phi_{1} p_{i} \frac{p_{j}^{a} e^{-\alpha \cdot d\left(x_{j}, x_{i}\right)}}{\sum_{j^{\prime}=1}^{N} p_{j^{\prime}}^{a} e^{-\alpha \cdot d\left(x_{j^{\prime}}, x_{i}\right)}},
$$

where $a \geq 0$. Equivalent behaviour is also defined for adversary $Q$. The subtraction of the terms $\phi_{1} p_{j}$ and $\phi_{2} q_{l}$ ensures that the total level of resource is conserved for each adversary.

The diagram in Figure 1 summarises the structure of the model, showing both within- and between-adversary effects. The internal flows represent the re-allocation of resources, varying according to distance and magnitude, while the external flows show how the threat emanating from a particular location is distributed. The flows shown are purely illustrative, and would depend on the parameter settings and spatial distribution in any realisation.

In addition to this, we provide a summary of notation in Table A.1 of Appendix A. It can be shown in a few steps that our spatially-explicit model (3) is a disaggregation of the original Richardson model (1). By defining $p:=\sum_{j=1}^{N} p_{j}$ and $q:=\sum_{l=1}^{M} q_{l}$ as the total hostility of each adversary, we prove in Appendix A that these total values satisfy the same relations as the original form.

\subsection{The within-adversary interaction term}

The inclusion of intra-adversarial effects - representing the reallocation of resources between the locations of an adversary - is one of the primary innovations of this model. It provides a mechanism by which 'support' can be provided from one location to another, thereby reflecting real-world strategic behaviour. For the entity $P$ at location $x_{j}$, this is represented in the model 
by the term

$$
\Pi\left(p_{j}\right):=-\phi_{1} p_{j}+\phi_{1} \sum_{i=1}^{N} p_{i} \frac{p_{j}^{a} e^{-\alpha \cdot d\left(x_{j}, x_{i}\right)}}{\sum_{j^{\prime}=1}^{N} p_{j^{\prime}}^{a} e^{-\alpha \cdot d\left(x_{j^{\prime}}, x_{i}\right)}} .
$$

The parameter $\alpha$ accounts for the effect of distance in impeding the reallocation of resources: larger values imply that movement is more costly, and that reallocation will therefore be biased towards shorter spatial ranges.

The parameter $a$, on the other hand, determines the effect of existing resource levels on reallocation behaviour. It can be interpreted in terms of return-to-scale: its value describes the extent to which allocation flows will be attracted towards locations which already have higher levels of resource. Here we distinguish two scenarios, representing distinct forms of behaviour:

(I) $a=0, \alpha>0$ : 'balancing' effect

The within-adversary action term simplifies to

$$
\Pi^{0}\left(p_{j}\right)=-\phi_{1} p_{j}+\phi_{1} \sum_{i=1}^{N} p_{i} \frac{e^{-\alpha \cdot d\left(x_{j}, x_{i}\right)}}{\sum_{j^{\prime}=1}^{N} e^{-\alpha \cdot d\left(x_{j^{\prime}}, x_{i}\right)}},
$$

that is, resources at location $x_{j}$ are re-distributed over the various locations of $P$ according to their distance from $x_{j}$. The inclusion of such term in the differential system has a balancing effect on the dynamics. This can be demonstrated for the case where $N=2$ : noting that $d\left(x_{i}, x_{i}\right)=0$ for $i=1,2$ and using the notation $d_{12}=d\left(x_{1}, x_{2}\right)=$ $d\left(x_{2}, x_{1}\right)$, the computations

$$
\begin{aligned}
\Pi^{0}\left(p_{1}\right) & =-\phi_{1} p_{1}+\phi_{1}\left(p_{1} \frac{1}{1+e^{-\alpha \cdot d_{12}}}+p_{2} \frac{e^{-\alpha \cdot d_{12}}}{e^{-\alpha \cdot d_{12}}+1}\right) \\
& =\frac{\phi_{1} e^{-\alpha \cdot d_{12}}}{1+e^{-\alpha \cdot d_{12}}}\left(p_{2}-p_{1}\right)
\end{aligned}
$$

show that $\Pi^{0}\left(p_{1}\right)$ is positive if $p_{1}<p_{2}$ and negative if $p_{1}>p_{2}$. We can also verify by numerical simulations that the system exhibits a similar 'balancing' effect for three or more locations per adversary; that is, the system

$$
\dot{p}_{j}=\Pi^{0}\left(p_{j}\right) \quad j=1,2,3, \ldots, N
$$

converges to an equilibrium $\left(p_{j}^{*}, j=1, \ldots, N\right)$ that satisfies $\left|p_{j}^{*}-p_{i}^{*}\right| \leq$ $\left|p_{j}(0)-p_{i}(0)\right|$ for all $i, j \in\{1, \ldots, N\}, i \neq j$. 
(II) $a>0, \alpha>0$ : 'rich-get-richer' effect

For simplicity we take $a=1$ to give the within-adversary action term

$$
\Pi^{1}\left(p_{j}\right)=-\phi_{1} p_{j}+\phi_{1} \sum_{i=1}^{N} p_{i} \frac{p_{j} e^{-\alpha \cdot d\left(x_{j}, x_{i}\right)}}{\sum_{j^{\prime}=1}^{N} p_{j^{\prime}} e^{-\alpha \cdot d\left(x_{j^{\prime}}, x_{i}\right)}}
$$

at location $x_{j}$ of adversary $P$. The impact of this bilinear term on the dynamics is quite different from that of the linear term $\Pi^{0}$ given above; resources are allocated proportionately to each location's existing resource level, resulting in a 'rich-get-richer' effect. For the $N=2$ case, again noting that $d\left(x_{i}, x_{i}\right)=0$ for $i=1,2$ and using $d_{12}$ to denote $d\left(x_{1}, x_{2}\right)=d\left(x_{2}, x_{1}\right)>0$, we find that

$$
\begin{aligned}
\Pi^{1}\left(p_{1}\right) & =-\phi_{1} p_{1}+\phi_{1}\left(p_{1} \frac{p_{1}}{p_{1}+p_{2} e^{-\alpha \cdot d_{12}}}+p_{2} \frac{p_{1} e^{-\alpha \cdot d_{12}}}{p_{1} e^{-\alpha \cdot d_{12}}+p_{2}}\right) \\
& =\frac{\phi_{1} p_{1}}{\left(p_{1}+p_{2} e^{-\alpha \cdot d_{12}}\right)\left(p_{1} e^{-\alpha \cdot d_{12}}+p_{2}\right)}\left(p_{2} e^{-\alpha \cdot d_{12}}\left(1-e^{-\alpha \cdot d_{12}}\right)\left(p_{1}-p_{2}\right)\right) .
\end{aligned}
$$

Using the fact that $e^{-\alpha \cdot d_{12}}<1$, we conclude that $\Pi^{1}\left(p_{1}\right)$ is positive if $p_{1}>p_{2}$ and negative if $p_{1}<p_{2}$ (note that this relation was just the opposite in the $a=0$ case). Our numerical simulations confirm that, for $N \geq 3$, escalation occurs at locations with the highest hostility levels while other sites with lower expenditure levels can further decrease; that is, there are $i, j \in\{1, \ldots, N\}, i \neq j$ such that $\left|p_{j}^{*}-p_{i}^{*}\right| \geq\left|p_{j}(0)-p_{i}(0)\right|$. In such as a situation, the reallocation effect results in the agglomeration of resources at a small number of locations.

\section{Model analysis}

Having introduced the model, we now present analysis of its dynamical properties. The primary motivation for this is to offer insight into the underlying social system; that is, findings that are interpretable in terms of conflict evolution. Issues such as stability, for example, have implications from the perspective of international relations: given that stability (i.e. the non-escalation of conflict) is a policy goal, establishing the conditions under which this occurs can offer real-world guidance. In addition to this, the novelty of the model means that such analysis is also of interest in itself, and may inform the application of the framework in other contexts. 
Since the model is not analytically tractable at higher dimensionality (i.e. for large values of $N$ and $M$ ), we focus in this section on lower-dimensional forms. These represent simplified cases, in terms of their spatial configuration, and allow the effect of the disaggregation to be explored at an elementary level. The question of whether these findings are also evident for more intricate configurations can subsequently be examined by numerical methods.

\subsection{Disaggregation for one actor $(N=2, M=1)$}

The simplest disaggregated case of the model is one in which one of the adversaries (taken here as $P$ ) is located at two sites (so that $N=2$ ), while the other is considered to be at only one location only (i.e. $M=1$ ), as in the aggregated form. This may correspond to a situation where one actor controls two non-contiguous territories, or where one simply possesses two military locations. Exploring this case allows us to isolate the effect of disaggregation on a single adversary, and we again consider separately the cases where $a$ is either zero or strictly positive.

\subsection{1. $a=0$}

We consider a simple spatial configuration in which $d\left(x_{1}, x_{2}\right)=1, d\left(x_{1}, y\right)=$ $d_{1}$, and $d\left(x_{2}, y\right)=d_{2}$. We take the parameter values $\sigma_{1}=\sigma_{2}, a=0$ and $b=1$ : substituting these and rescaling the system (3), we arrive at

$$
\begin{aligned}
\dot{p}_{1} & =-p_{1}-\phi_{1} p_{1}+\phi_{1} \frac{p_{1}+p_{2} e^{-\alpha}}{1+e^{-\alpha}}+\rho_{1} \frac{q p_{1} e^{-\beta d_{1}}}{p_{1} e^{-\beta d_{1}}+p_{2} e^{-\beta d_{2}}}+\frac{\epsilon_{1}}{2}, \\
\dot{p}_{2} & =-p_{2}-\phi_{1} p_{2}+\phi_{1} \frac{p_{1} e^{-\alpha}+p_{2}}{1+e^{-\alpha}}+\rho_{1} \frac{q p_{2} e^{-\beta d_{2}}}{p_{1} e^{-\beta d_{1}}+p_{2} e^{-\beta d_{2}}}+\frac{\epsilon_{1}}{2}, \\
\dot{q} & =\rho_{2}\left(p_{1}+p_{2}\right)-q-\phi_{2} q+\phi_{2} q+\epsilon_{2} .
\end{aligned}
$$

We introduce a new variable $r=p_{1}-p_{2}$ for the difference between hostility levels of the two locations of $P$ (in real world terms, this represents the differential between an actor's power at its primary sites). Using this, the above system transforms into

$$
\begin{aligned}
& \dot{p}=-p+\rho_{1} q+\epsilon_{1} \\
& \dot{q}=\rho_{2} p-q+\epsilon_{2}, \\
& \dot{r}=-r-\phi_{1} r+\phi_{1} r \frac{\left(1-e^{-\alpha}\right)}{\left(1+e^{-\alpha}\right)}+\rho_{1} q \frac{\left(1-e^{-\beta\left(d_{2}-d_{1}\right)}\right) p+\left(1+e^{-\beta\left(d_{2}-d_{1}\right)}\right) r}{\left(1+e^{-\beta\left(d_{2}-d_{1}\right)}\right) p+\left(1-e^{-\beta\left(d_{2}-d_{1}\right)}\right) r} .
\end{aligned}
$$


The equations for $\dot{p}$ and $\dot{q}$ decouple and formulate the system (1), the dynamics of which are well understood: all solutions converge to the equilibrium $(\bar{p}, \bar{q})$ given in $(2)$ if $\rho_{1} \rho_{2}<1$. Therefore for our analysis of system (4) we assume that this condition holds, and consider only the last equation of (4) under the constraint that $p$ and $q$ are at their equilibria. This way a single limiting equation of the system (4) arises:

$$
\dot{r}=-r \frac{1+e^{-\alpha}+2 \phi_{1} e^{-\alpha}}{1+e^{-\alpha}}+\rho_{1} \bar{q} \frac{\left(1-e^{-\beta\left(d_{2}-d_{1}\right)}\right) \bar{p}+\left(1+e^{-\beta\left(d_{2}-d_{1}\right)}\right) r}{\left(1+e^{-\beta\left(d_{2}-d_{1}\right)}\right) \bar{p}+\left(1-e^{-\beta\left(d_{2}-d_{1}\right)}\right) r} .
$$

We show in Appendix A that the system converges to a stable equilibrium under the same conditions to those which apply for the original Richardson model. For non-trivial spatial configurations, however, the resource levels at equilibrium are unequal across the locations of the disaggregated actor: higher volume is accumulated at the site that is closer to its adversary. This occurs as a result of the action-reaction mechanism: more threat is experienced at this site, and resources are therefore accumulated at a higher rate.

\subsection{2. $a>0$}

The case where $a>0$ involves the reallocation of resources in proportion to existing resource levels. We set $a=1$, and take other parameters as in the previous section: $\sigma_{1}=\sigma_{2}, b=1, d\left(x_{1}, x_{2}\right)=1, d\left(x_{1}, y\right)=d_{1}$, and $d\left(x_{2}, y\right)=d_{2}$. Rescaling the system (3) and again considering the variable $r=p_{1}-p_{2}$, we arrive at

$$
\begin{aligned}
\dot{p}= & -p+\rho_{1} q+\epsilon_{1}, \\
\dot{q}= & \rho_{2} p-q+\epsilon_{2}, \\
\dot{r}= & -r-\phi_{1} r+\rho_{1} q \frac{\left(1-e^{-\beta\left(d_{2}-d_{1}\right)}\right) p+\left(1+e^{-\beta\left(d_{2}-d_{1}\right)}\right) r}{\left(1+e^{-\beta\left(d_{2}-d_{1}\right)}\right) p+\left(1-e^{-\beta\left(d_{2}-d_{1}\right)}\right) r} \\
& +\frac{\phi_{1}}{2}(p+r) \frac{\left(1-e^{-\alpha}\right) p+\left(1+e^{-\alpha}\right) r}{\left(1+e^{-\alpha}\right) p+\left(1-e^{-\alpha}\right) r}+\frac{\phi_{1}}{2}(p-r) \frac{\left(e^{-\alpha}-1\right) p+\left(1+e^{-\alpha}\right) r}{\left(1+e^{-\alpha}\right) p+\left(e^{-\alpha}-1\right) r} .
\end{aligned}
$$

As in the $a=0$ case, the equations for $\dot{p}$ and $\dot{q}$ decouple and formulate the system (1), for which all solutions converge to the equilibrium $(\bar{p}, \bar{q})$ given in (2) if $\rho_{1} \rho_{2}<1$. Again, therefore, we assume that this last inequality holds, and consider only the last equation of (5) under the constraint that $p$ and $q$ are at their equilibria. A single limiting equation of the system (5) again 
arises in this case:

$$
\begin{aligned}
\dot{r}= & -r-\phi_{1} r+\rho_{1} \bar{q} \frac{\left(1-e^{-\beta\left(d_{2}-d_{1}\right)}\right) \bar{p}+\left(1+e^{-\beta\left(d_{2}-d_{1}\right)}\right) r}{\left(1+e^{-\beta\left(d_{2}-d_{1}\right)}\right) \bar{p}+\left(1-e^{-\beta\left(d_{2}-d_{1}\right)}\right) r} \\
& +\frac{\phi_{1}}{2}(\bar{p}+r) \frac{\left(1-e^{-\alpha}\right) \bar{p}+\left(1+e^{-\alpha}\right) r}{\left(1+e^{-\alpha}\right) \bar{p}+\left(1-e^{-\alpha}\right) r}+\frac{\phi_{1}}{2}(\bar{p}-r) \frac{\left(e^{-\alpha}-1\right) \bar{p}+\left(1+e^{-\alpha}\right) r}{\left(1+e^{-\alpha}\right) \bar{p}+\left(e^{-\alpha}-1\right) r} .
\end{aligned}
$$

Using numerical simulations, we can confirm that there is an equilibrium of the limiting equation that attracts every solution in the feasible region $(|r(0)| \leq \bar{p})$. This equilibrium has larger value than the globally stable steady state $\bar{r}_{+}$obtained for $a=0$. This finding also highlights the difference between the characteristics of the two scenarios ' $a=0$ ' and ' $a>0$ '; while the former represents a strategy by which an actor seeks to lessen the differences between the levels of hostility at its various locations (a 'balancing' effect), the latter favours agglomeration and drives the system away from such balanced state ('rich-get-richer' effect). Although the stability of the aggregated system is unchanged, behaviour of this type leads to a more spatially-concentrated distribution of arms, which could be interpreted as a more precarious situation from the perspective of security.

An alternative perspective on these results concerns the behaviour of an actor which is seeking to maintain a prescribed division of resources between its locations. A feasible real-world policy, for example, might dictate that levels at one location are maintained at a given percentage of the adversary's total: this behaviour is neither equalising nor agglomerative. In such cases, parameters can be tuned in order to achieve this (given the other adversary's distribution), and the values required to do this can be interpreted in behavioural terms. The higher value of $a$ required to achieve the prescribed balance, for example, the more such a strategy requires active and positive re-allocation, relative to a purely equitable strategy.

\subsection{Disaggregation for both actors $(N=2, M=2)$}

A more complex version of the model involves the disaggregation of both actors: both $P$ and $Q$ are assumed to be located at two sites. This is the simplest version of the model for which internal spatial dynamics are present for both actors. This scenario can again be interpreted as representing two actors which both have presence in two unconnected territories, or simply that both possess two resource locations (which might be military bases, for example). 


\subsection{1. $a=0$}

To consider the effect of balancing behaviour in this case, we consider a simplified spatial configuration. In particular, we consider a scenario in which $d\left(x_{1}, x_{2}\right)=d\left(y_{1}, y_{2}\right)=1, d\left(x_{1}, y_{1}\right)=d\left(x_{2}, y_{2}\right)=0$, and $d\left(x_{1}, y_{2}\right)=$ $d\left(x_{2}, y_{1}\right)=1$ : the system contains only two locations, one unit apart, at which both adversaries are located. Taking these values, setting $a=0, b=1$ and $\sigma_{1}=\sigma_{2}$, and rescaling the system (3), we arrive at

$$
\begin{aligned}
& \dot{p}_{1}=-p_{1}-\phi_{1} p_{1}+\phi_{1} \frac{p_{1}+p_{2} e^{-\alpha}}{1+e^{-\alpha}}+\rho_{1}\left(\frac{q_{1} p_{1}}{p_{1}+p_{2} e^{-\beta}}+\frac{q_{2} p_{1} e^{-\beta}}{p_{1} e^{-\beta}+p_{2}}\right)+\frac{\epsilon_{1}}{2}, \\
& \dot{p}_{2}=-p_{2}-\phi_{1} p_{2}+\phi_{1} \frac{p_{1} e^{-\alpha}+p_{2}}{1+e^{-\alpha}}+\rho_{1}\left(\frac{q_{1} p_{2} e^{-\beta}}{p_{1}+p_{2} e^{-\beta}}+\frac{q_{2} p_{2}}{p_{1} e^{-\beta}+p_{2}}\right)+\frac{\epsilon_{1}}{2}, \\
& \dot{q}_{1}=\rho_{2}\left(\frac{p_{1} q_{1}}{q_{1}+q_{2} e^{-\beta}}+\frac{p_{2} q_{1} e^{-\beta}}{q_{1} e^{-\beta}+q_{2}}\right)-q_{1}-\phi_{2} q_{1}+\phi_{2} \frac{q_{1}+q_{2} e^{-\alpha}}{1+e^{-\alpha}}+\frac{\epsilon_{2}}{2}, \\
& \dot{q}_{2}=\rho_{2}\left(\frac{p_{1} q_{2} e^{-\beta}}{q_{1}+q_{2} e^{-\beta}}+\frac{p_{2} q_{2}}{q_{1} e^{-\beta}+q_{2}}\right)-q_{2}-\phi_{2} q_{2}+\phi_{2} \frac{q_{1} e^{-\alpha}+q_{2}}{1+e^{-\alpha}}+\frac{\epsilon_{2}}{2} .
\end{aligned}
$$

We introduce new variables $p=p_{1}+p_{2}, r=p_{1}-p_{2}, q=q_{1}+q_{2}, s=q_{1}-q_{2}$, and transform the above system into

$$
\begin{aligned}
\dot{p}= & -p+\rho_{1} q+\epsilon_{1}, \\
\dot{q}= & \rho_{2} p-q+\epsilon_{2}, \\
\dot{r}= & -r-\phi_{1} r+\phi_{1} r \frac{\left(1-e^{-\alpha}\right)}{\left(1+e^{-\alpha}\right)}+\frac{\rho_{1}}{2}(q+s) \frac{\left(1-e^{-\beta}\right) p+\left(1+e^{-\beta}\right) r}{\left(1+e^{-\beta}\right) p+\left(1-e^{-\beta}\right) r} \\
& +\frac{\rho_{1}}{2}(q-s) \frac{\left(e^{-\beta}-1\right) p+\left(1+e^{-\beta}\right) r}{\left(1+e^{-\beta}\right) p+\left(e^{-\beta}-1\right) r}, \\
\dot{s}= & -s-\phi_{2} s+\phi_{2} s \frac{\left(1-e^{-\alpha}\right)}{\left(1+e^{-\alpha}\right)}+\frac{\rho_{2}}{2}(p+r) \frac{\left(1-e^{-\beta}\right) q+\left(1+e^{-\beta}\right) s}{\left(1+e^{-\beta}\right) q+\left(1-e^{-\beta}\right) s} \\
& +\frac{\rho_{2}}{2}(p-r) \frac{\left(e^{-\beta}-1\right) q+\left(1+e^{-\beta}\right) s}{\left(1+e^{-\beta}\right) q+\left(e^{-\beta}-1\right) s} .
\end{aligned}
$$

As in the cases shown in Section 3.1, the equations for $\dot{p}$ and $\dot{q}$ decouple and reduce to the original Richardson system (1). Since it is known that all solutions converge to the equilibrium $(\bar{p}, \bar{q})$ if $\rho_{1} \rho_{2}<1$, we therefore assume that this inequality holds, and consider only the last two equations of (6) under the constraint that $p$ and $q$ are at their equilibria. In this way, we find a limiting system of (6) that consists of two equations only. 
We investigate the stability of the trivial equilibrium $(0,0)$ in the limiting system by linearising around $(0,0)$. At this equilibrium, hostility is evenly distributed over the locations of an adversary hence we can say the system is at a balanced state and we find $\left(\bar{p}_{1}, \bar{p}_{2}, \bar{q}_{1}, \bar{q}_{2}\right)=(\bar{p} / 2, \bar{p} / 2, \bar{q} / 2, \bar{q} / 2)$. Linearisation yields

$$
\left(\begin{array}{l}
\dot{r} \\
\dot{s}
\end{array}\right)=J_{1}\left(\begin{array}{l}
r \\
s
\end{array}\right)
$$

where $J_{1}$ denotes the Jacobian,

$$
J_{1}=\left(\begin{array}{cc}
-1-\phi_{1}+\phi_{1} \omega+\rho_{1}\left(1-\eta^{2}\right) & \rho_{1} \eta \\
\rho_{2} \eta & -1-\phi_{2}+\phi_{2} \omega+\rho_{2}\left(1-\eta^{2}\right)
\end{array}\right),
$$

with

$$
\omega=\frac{1-e^{-\alpha}}{1+e^{-\alpha}}, \quad \eta=\frac{1-e^{-\beta}}{1+e^{-\beta}} .
$$

We observe that $\omega$ is monotonically increasing in $\alpha$, with $\omega=0$ at $\alpha=0$ and $\omega \rightarrow 1$ as $\alpha \rightarrow \infty$. Similarly, $\eta$ is monotonically increasing in $\beta$, with $\eta=0$ at $\beta=0$ and $\eta \rightarrow 1$ as $\beta \rightarrow \infty$. Thus for non-negative $\alpha$ and $\beta$ it holds that $0 \leq \omega, \eta<1$.

We examine by mathematical analysis the dependence of stability on various model parameters, with an emphasis on the support parameters $\phi_{1}$ and $\phi_{2}$. The main result of this section is stated below, while its proof is presented in Appendix A.

Theorem 1. If $\rho_{2}\left(1-\eta^{2}\right) \geq 1+\phi_{2}(1-\omega)$ then the trivial equilibrium $(\bar{r}, \bar{s})=$ $(0,0)$ is unstable for all values of $\phi_{1}$ and $\rho_{1}$. If $\rho_{2}\left(1-\eta^{2}\right)<1+\phi_{2}(1-\omega)$ then stability depends on the value of $\phi_{1}$ and $\rho_{1}$, more precisely there is a threshold quantity

$$
\mathcal{T}_{1}=\frac{1}{1+\phi_{1}(1-\omega)}\left(\rho_{1}\left(1-\eta^{2}\right)+\frac{\rho_{1} \rho_{2} \eta^{2}}{1+\phi_{2}(1-\omega)-\rho_{2}\left(1-\eta^{2}\right)}\right)
$$

such that the trivial equilibrium $(\bar{r}, \bar{s})=(0,0)$ is unstable if $\mathcal{T}_{1}>1$ and stable if $\mathcal{T}_{1}<1$. A critical value for stability, $\tilde{\phi}_{1}$, is defined as

$$
\tilde{\phi}_{1}=\left(\rho_{1}\left(1-\eta^{2}\right)+\frac{\rho_{1} \rho_{2} \eta^{2}}{1+\phi_{2}(1-\omega)-\rho_{2}\left(1-\eta^{2}\right)}-1\right)(1-\omega)^{-1},
$$

and the trivial solution is unstable if $\phi_{1}<\tilde{\phi}_{1}$, but stabilises as $\phi_{1}$ exceeds $\tilde{\phi}_{1}$. Similar relations can be obtained in terms of $\phi_{2}$. 
This result can also be interpreted in terms of real-world behaviour. While the conditions for stability in an aggregated sense are identical to those for the original model, interesting behaviour arises with respect to the spatial distribution of resources. The equilibrium $(\bar{r}, \bar{s})=(0,0)$ that we investigated corresponds to the equitable distribution of resources between the two locations. Our analysis reveals a stability condition involving $\rho_{1}, \rho_{2}, \phi_{1}$ and $\phi_{2}$ implying that resource reallocation has a material effect on the tendency of the system to concentrate at one location. In particular, it is shown that increasing $\phi_{1}$ and $\phi_{2}$ (that is, increasing the reallocation effect) expands the stability region (see Figure 2a). This effect therefore acts as a counterweight to the tendency of the system to evolve towards a potentially insecure situation in which resources are concentrated in one location.

\subsection{2. $a>0$}

We also investigate the form of the model incorporating positive feedback in resource allocation. We do this for the same spatial configuration as in the previous section: $d\left(x_{1}, x_{2}\right)=d\left(y_{1}, y_{2}\right)=1, d\left(x_{1}, y_{1}\right)=d\left(x_{2}, y_{2}\right)=0$, and $d\left(x_{1}, y_{2}\right)=d\left(x_{2}, y_{1}\right)=1$. With $a=b=1, \sigma_{1}=\sigma_{2}$, and variables $p=p_{1}+p_{2}$, $r=p_{1}-p_{2}, q=q_{1}+q_{2}, s=q_{1}-q_{2}$ the rescaled system (3) becomes

$$
\begin{aligned}
\dot{p}= & -p+\rho_{1} q+\epsilon_{1}, \\
\dot{q}= & \rho_{2} p-q+\epsilon_{2}, \\
\dot{r}= & -r-\phi_{1} r+\frac{\phi_{1}}{2}(p+r) \frac{\left(1-e^{-\alpha}\right) p+\left(1+e^{-\alpha}\right) r}{\left(1+e^{-\alpha}\right) p+\left(1-e^{-\alpha}\right) r} \\
& +\frac{\phi_{1}}{2}(p-r) \frac{\left(e^{-\alpha}-1\right) p+\left(1+e^{-\alpha}\right) r}{\left(1+e^{-\alpha}\right) p+\left(e^{-\alpha}-1\right) r}+\frac{\rho_{1}}{2}(q+s) \frac{\left(1-e^{-\beta}\right) p+\left(1+e^{-\beta}\right) r}{\left(1+e^{-\beta}\right) p+\left(1-e^{-\beta}\right) r} \\
& +\frac{\rho_{1}}{2}(q-s) \frac{\left(e^{-\beta}-1\right) p+\left(1+e^{-\beta}\right) r}{\left(1+e^{-\beta}\right) p+\left(e^{-\beta}-1\right) r}, \\
\dot{s}= & -s-\phi_{2} s+\frac{\phi_{2}}{2}(q+s) \frac{\left(1-e^{-\alpha}\right) q+\left(1+e^{-\alpha}\right) s}{\left(1+e^{-\alpha}\right) q+\left(1-e^{-\alpha}\right) s} \\
& +\frac{\phi_{2}}{2}(q-s) \frac{\left(e^{-\alpha}-1\right) q+\left(1+e^{-\alpha}\right) s}{\left(1+e^{-\alpha}\right) q+\left(e^{-\alpha}-1\right) s}+\frac{\rho_{2}}{2}(p+r) \frac{\left(1-e^{-\beta}\right) q+\left(1+e^{-\beta}\right) s}{\left(1+e^{-\beta}\right) q+\left(1-e^{-\beta}\right) s} \\
& +\frac{\rho_{2}}{2}(p-r) \frac{\left(e^{-\beta}-1\right) q+\left(1+e^{-\beta}\right) s}{\left(1+e^{-\beta}\right) q+\left(e^{-\beta}-1\right) s} .
\end{aligned}
$$

The equations for $\dot{p}$ and $\dot{q}$ decouple as before, and so we assume that $\rho_{1} \rho_{2}<1$ and consider only the last two equations of (7) under the constraint that $p$ 


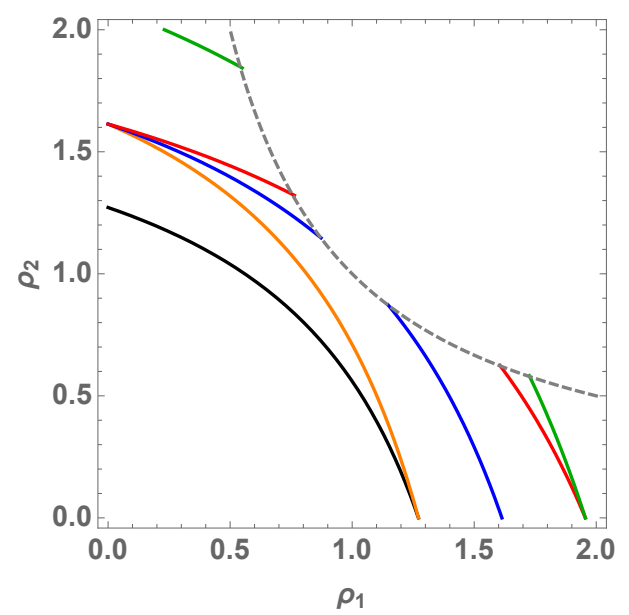

(a) $a=0$

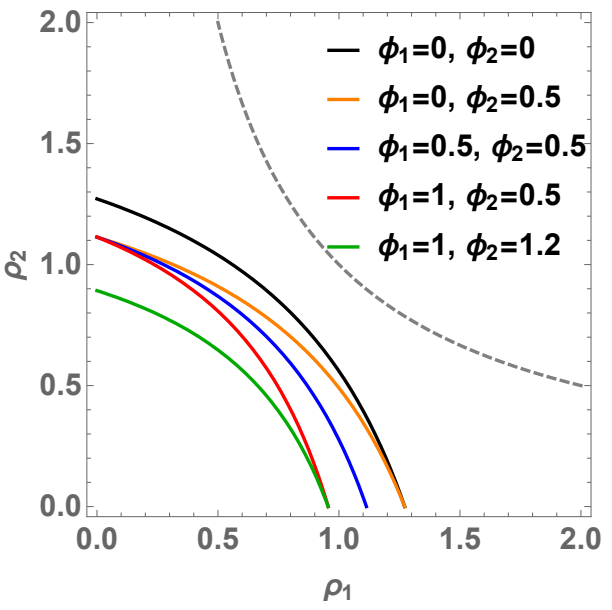

(b) $a=1$

Figure 2: Illustration of stability boundaries in the models (6) (Figure 2a) and (7) (Figure $2 \mathrm{~b}$ ) for different values of $\phi_{1}$ and $\phi_{2}$. Figure 2a: For each curve, the trivial solution $(\bar{r}, \bar{s})=(0,0)$ is stable for all values of $\left(\rho_{1}, \rho_{2}\right)$ below the curve. In this case, increasing $\phi_{1}$ and $\phi_{2}$ expands the stability region. Figure 2b: For each curve, the trivial solution $(\bar{r}, \bar{s})=(0,0)$ is unstable for all values of $\left(\rho_{1}, \rho_{2}\right)$ below the curve. Increasing $\phi_{1}$ and $\phi_{2}$ reduces the stability region. The other parameters are: $\epsilon_{1}=\epsilon_{2}=0.1, \beta=1, \alpha=1$. The dashed grey line marks $\rho_{1} \rho_{2}=1$.

and $q$ are at their equilibria, $(\bar{p}, \bar{q})$. Using this approach, we find a limiting system of (7) that consists of two equations only.

We are interested in the stability of the trivial equilibrium $(\bar{r}, \bar{s})=(0,0)$ in the limiting system. Recalling that $r=p_{1}-p_{2}$ and $s=q_{1}-q_{2}$, the steady state at each location is given as $\left(\bar{p}_{1}, \bar{p}_{2}, \bar{q}_{1}, \bar{q}_{2}\right)=(\bar{p} / 2, \bar{p} / 2, \bar{q} / 2, \bar{q} / 2)$. By linearising the limiting system around $(0,0)$, we derive

$$
\left(\begin{array}{l}
\dot{r} \\
\dot{s}
\end{array}\right)=J_{2}\left(\begin{array}{l}
r \\
s
\end{array}\right),
$$

where the Jacobian is given as

$$
J_{2}=\left(\begin{array}{cc}
-1+\phi_{1} \omega(1-\omega)+\rho_{1}\left(1-\eta^{2}\right) & \rho_{1} \eta \\
\rho_{2} \eta & -1+\phi_{2} \omega(1-\omega)+\rho_{2}\left(1-\eta^{2}\right)
\end{array}\right) .
$$

The following theorem summarises stability properties in system (7), and its proof is given in Appendix A. 


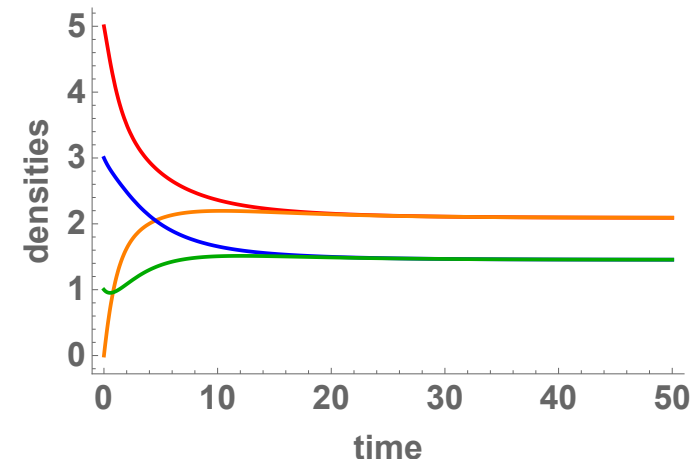

(a) $a=0$

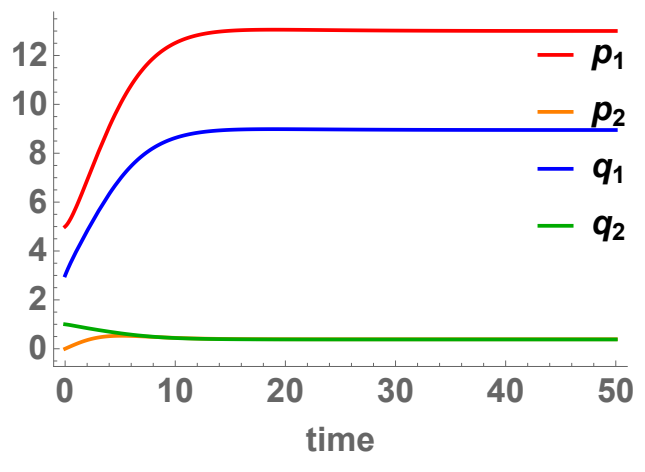

(b) $a=1$

Figure 3: Solution curves in the models (6) (Figure 3a) and (7) (Figure 3b) when $\phi_{1}=1$, $\phi_{2}=0.5$. The difference between the two scenarios ' $a=0$ ' (Figure (a)) and ' $a=1$ ' (Figure (b)) in the stability of the balanced equilibrium $(\bar{r}, \bar{s})=(0,0) \Leftrightarrow \bar{p}_{1}=\bar{p}_{2}, \bar{q}_{1}=\bar{q}_{2}$ is demonstrated. The other parameters are: $\epsilon_{1}=\epsilon_{2}=0.4, \beta=1, \alpha=1$, and $\rho_{1}=1.3$, $\rho_{2}=0.6$ therefore $\rho_{1} \rho_{2}<1$.

Theorem 2. If $\phi_{2} \omega(1-\omega)+\rho_{2}\left(1-\eta^{2}\right)>1$ then the trivial equilibrium $(\bar{r}, \bar{s})=(0,0)$ is unstable for all values of $\phi_{1}$ and $\rho_{1}$. If $\phi_{2} \omega(1-\omega)+\rho_{2}(1-$ $\left.\eta^{2}\right)<1$ then stability depends on the value of $\phi_{1}$ and $\rho_{1}$. More precisely, there is a threshold quantity

$$
\mathcal{T}_{2}=\phi_{1} \omega(1-\omega)+\rho_{1}\left(1-\eta^{2}\right)+\frac{\rho_{1} \rho_{2} \eta^{2}}{1-\phi_{2} \omega(1-\omega)-\rho_{2}\left(1-\eta^{2}\right)}
$$

such that the trivial equilibrium $(\bar{r}, \bar{s})=(0,0)$ is unstable if $\mathcal{T}_{2}>1$ and locally asymptotically stable if $\mathcal{T}_{2}<1$. A critical value $\breve{\phi}_{1}$ for stability is defined as

$$
\breve{\phi}_{1}=\left(1-\rho_{1}\left(1-\eta^{2}\right)-\frac{\rho_{1} \rho_{2} \eta^{2}}{1-\phi_{2} \omega(1-\omega)-\rho_{2}\left(1-\eta^{2}\right)}\right) \omega^{-1}(1-\omega)^{-1},
$$

and the trivial solution is locally asymptotically stable for $\phi_{1}<\breve{\phi}_{1}$, but it loses its stability as $\phi_{1}$ increases through $\breve{\phi}_{1}$. Similar relations can be obtained in terms of $\phi_{2}$.

In this case, therefore, the reallocation mechanism has the opposite effect to that when it was purely distance-based (see Figure 2b, increasing reallocation effect decreases the stability region). When $a>0$, this encodes a 'rich-get-richer' effect, which merely magnifies the effect of inter-adversary 
hostility (the action-reaction term) in concentrating activity in one location. The reallocation follows the growth stimulated by antagonism (with resources being bolstered at the locations where threat is experienced) and therefore reinforces the agglomeration in these locations. If it is assumed that such sites represent potential conflict flashpoints, then this positive feedback effect results in increased risk. Analytical results of this and the previous sections are illustrated in Figures 2 and 3, which also highlight the difference between the two scenarios ' $a=0$ ': balancing effect and ' $a=0$ ': 'rich-get-richer' effect.

In the remainder of our analysis we investigate whether the two support parameters $\phi_{1}$ and $\phi_{2}$ can jointly control stability.

Theorem 3. It is possible to obtain a threshold quantity $\mathcal{T}_{3}$ such that the trivial equilibrium is locally asymptotically stable if $\mathcal{T}_{3}<1$, and unstable if $\mathcal{T}_{3}>1$. We define

$$
\phi_{1}^{*}=\frac{\phi_{1}}{\mathcal{T}_{3}}+\frac{\rho_{1}\left(1-\eta^{2}\right)}{\omega(1-\omega)}\left(\frac{1}{\mathcal{T}_{3}}-1\right), \quad \phi_{2}^{*}=\frac{\phi_{2}}{\mathcal{T}_{3}}+\frac{\rho_{2}\left(1-\eta^{2}\right)}{\omega(1-\omega)}\left(\frac{1}{\mathcal{T}_{3}}-1\right) .
$$

If $\mathcal{T}_{3}<1$ then increasing $\phi_{1}$ to $\phi_{1}^{*}$ and $\phi_{2}$ to $\phi_{2}^{*}$ destabilises the trivial equilibrium. If $\mathcal{T}_{3}>1$ then stabilising the trivial equilibrium is possible if $\mathcal{T}_{3} \leq 1+\phi_{1} \omega(1-\omega) /\left(\rho_{1}\left(1-\eta^{2}\right)\right)$ and $\mathcal{T}_{3} \leq 1+\phi_{2} \omega(1-\omega) /\left(\rho_{2}\left(1-\eta^{2}\right)\right)$ hold, by decreasing $\phi_{1}$ to $\phi_{1}^{*}$ and $\phi_{2}$ to $\phi_{2}^{*}$. Otherwise it is not possible to stabilise the trivial equilibrium by changing only $\phi_{1}$ and $\phi_{2}$.

The proof of this theorem has been moved to Appendix A. From the perspective of real-world conflict, this result can be interpreted in terms of conflict prevention. Manipulations of $\phi_{1}$ and $\phi_{2}$ represent feasible policy interventions that may be applied: either restrictions on the movement of resources, or the encouragement of movement towards under-resourced areas. The analysis above indicates that such changes can, in certain circumstances, control the stability of the system, thereby suggesting that they represent a meaningful policy intervention. It is also true, however, that such changes will not be effective in all circumstances, which implies that other interventions (corresponding to the other parameters in the model) may also be necessary in such cases.

\section{A numerical example}

For reasons of tractability, our mathematical analysis has been restricted to low-dimensional forms of the model. We have established a number of 
properties in these basic scenarios: in particular, the influence that the reallocation term has in affecting the spatial distribution of resources at equilibrium. These simplified examples were intended to isolate the effects of disaggregation (and allow them to be investigated analytically), which we would expect to be manifested in more complex, higher-dimensional scenarios. In this section, we carry out a number of numerical simulations to examine the behaviour of the model in such scenarios, and in particular to verify that the results of our analytical work remain valid in these configurations.

In terms of its real-world relevance, the model is intended to represent situations in which two actors (most immediately nation-states) are in conflict in some large spatial territory. The locations over which their resources are distributed are intended to represent areas with significant military value, such as bases or large cities. A realistic simulation, therefore, will typically involve both adversaries having numbers of locations of the order of tens, and it is such situations that we examine here.

Figure 4 depicts four scenarios that are derived by varying the support parameters $\phi$ and $a$. The spatial configuration of the two adversaries is the same in all cases, and the two sides are distinguished by colour, so that the blue points are adversaries to the green.

The spatial configuration is derived as follows: a set of 50 points in the unit square, given by $\mathcal{M}=\{(x, y) \mid 0 \leq x \leq 1,0 \leq y \leq 1\}$, are uniformly randomly generated. We suppose that each adversary has an associated territory and so points with $x \leq 0.5$ are initially allocated to the green group and points with $x>0.5$ are initially allocated to the blue group. However, in order to examine the role that support might play in conflict scenarios, we then reallocate some of the points within each territory to the opposing side. This enables us to examine the way in which locations that are surrounded by enemy territory can be supported by the other locations of the same adversary. Specifically, we let those points lying in the region $\{(x, y) \mid 0.2 \leq x \leq 0.4,0.4 \leq y \leq 0.6\}$ convert to blue and let those points lying in the region $\{(x, y) \mid 0.6 \leq x \leq 0.8,0.4 \leq y \leq 0.6\}$ convert to green. This produces two opposing 'islands' within the main territories dominated by each side: the subfigures in Figure 4 show the spatial configuration that resulted from one such realisation of this process.

For each parameter configuration, the model in equation (3) is numerically solved using the 4th order Runge-Kutta procedure, using step sizes of $\delta t=$ 0.1. An equilibrium is detected when $\left|p_{j}(t+\delta t)-p_{j}(t)\right| /\left|p_{j}(t)\right|<10^{-5}$ and $\left|q_{l}(t+\delta t)-q_{l}(t)\right| /\left|q_{l}(t)\right|<10^{-5}$ for all $j=1,2, \ldots, N$ and $l=1,2, \ldots, M$. 


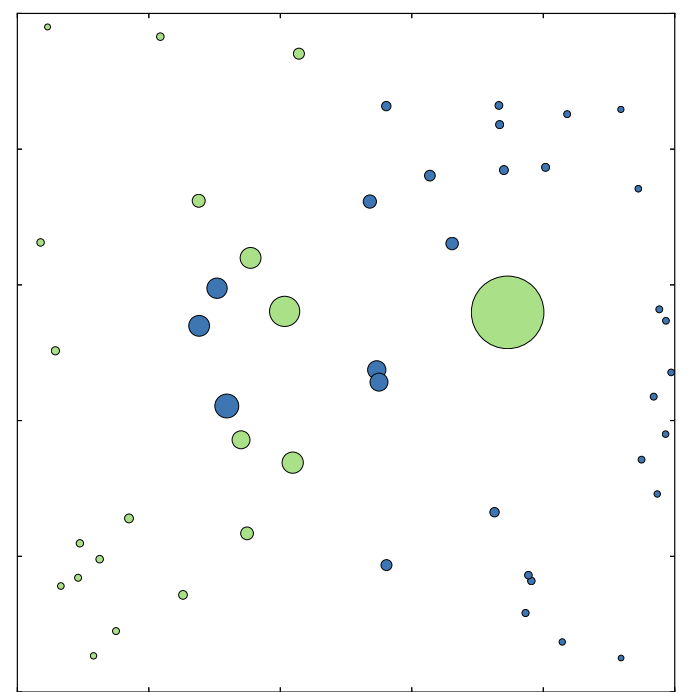

(a) $\phi=0$

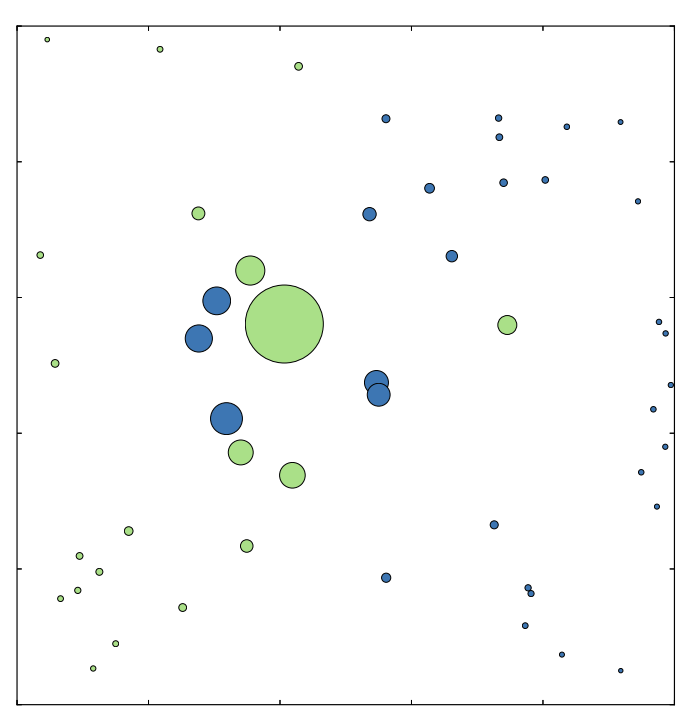

(c) $\phi=0.5, a=1$

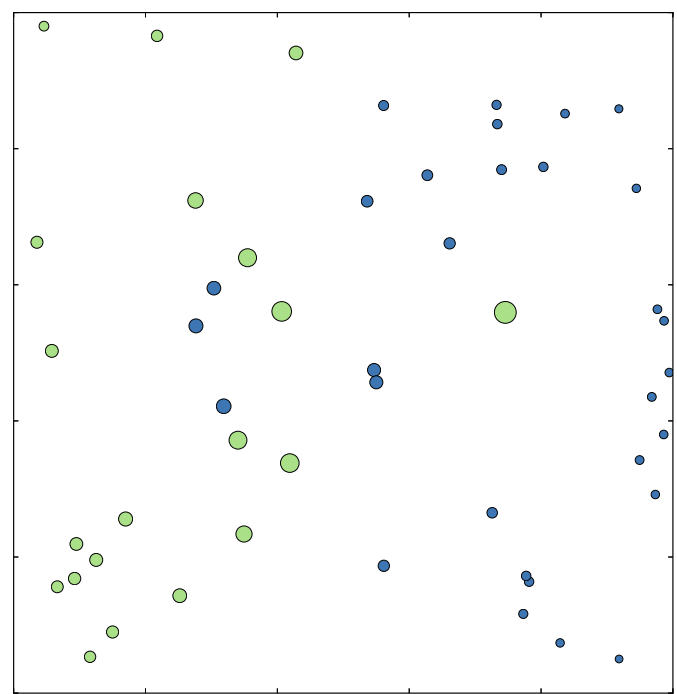

(b) $\phi=0.5, a=0$

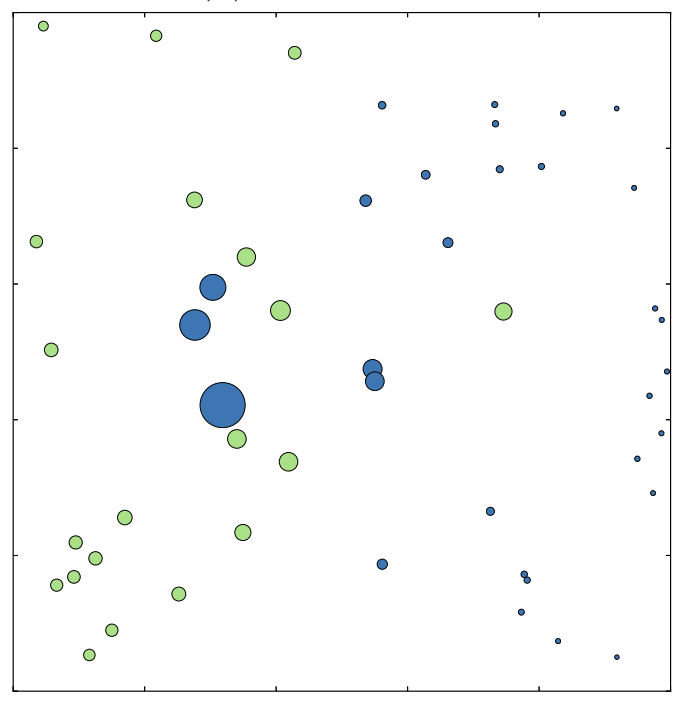

(d) $\phi=0.5, a=0$ for green, $a=1$ for blue

Figure 4: Equilibrium values for a range of conflict scenarios in which two adversaries (green and blue) are distributed over a two-dimensional square. Each panel represents a run of the model for given values of $\phi$ and $a$, with the other parameters held constant as follows: $\sigma_{1}=\sigma_{2}=1, \epsilon_{1}=\epsilon_{2}=0.1, \beta=1, \alpha=1, \rho_{1}=\rho_{2}=0.8$. In each case, the size of the point indicates the level of resources (i.e. $p_{j}$ and $q_{l}$ ) at equilibrium. 
In Figure 4a, no support dynamics are included (as indicated by $\phi=0$ ). Using the size of each point to represent the value of the resulting equilibrium, we see that those locations in enemy territory are typically those that are subject to the greatest threat and therefore have the largest resource levels. This is particularly the case for the green adversary, which possesses just one location in enemy territory.

In Figure 4b, the support term is included in the model with $a=0$. The resulting equilibrium is more evenly distributed than in Figure 4a, as resources can now be re-distributed between the locations of an adversary. In particular, in this scenario, the resource levels of those locations in enemy territory are distributed more evenly across the adversary's other locations. Those locations on the outer boundary of the space 'support' those in the middle, reducing the total amount of hostility at that location and serving to dampen the system as a whole.

In Figure 4c, the support term is included with $a=1$. In this case, we see a more uneven distribution of equilibrium values in comparison to both Figure $4 \mathrm{~b}$ and, to a lesser extent, Figure 4a. This supports the findings in Sections 3 , which found that $a>0$ leads to a phenomenon by which locations with little threat upon them will share their resources within their group by sending them towards those locations that need them most. In Figure 4c, we also observe an important distinction from Figure 4a: it is not the location within enemy territory that results in the largest hostility; but, instead, a location within green territory, which is closer to the majority of other green locations. We posit that this difference results from the ease with which the green side can share resources within its own territory. In essence, the green location furthest to the right and in blue territory is so far into enemy territory that it cannot receive support.

In Figure $4 \mathrm{~d}$, we again take $\phi=0.5$, but in this case each adversary is assigned a different value of $a$ : we will use $a_{b}$ to denote the value used for blue's behaviour, and $a_{g}$ for green. Here we take $a_{g}=0$ and $a_{b}=$ 1: for such a scenario, we observe that the green locations, which are reallocating resources all their locations, have hostility levels that are more evenly distributed than the blue locations, which are sending resources to those locations within enemy territory. The value of $a$ in real world conflict scenarios is likely to require a strategic decision by actors in the system. An adversary may choose to either distribute its resources more evenly among its different locations or may instead choose to send resources where they are needed most. 


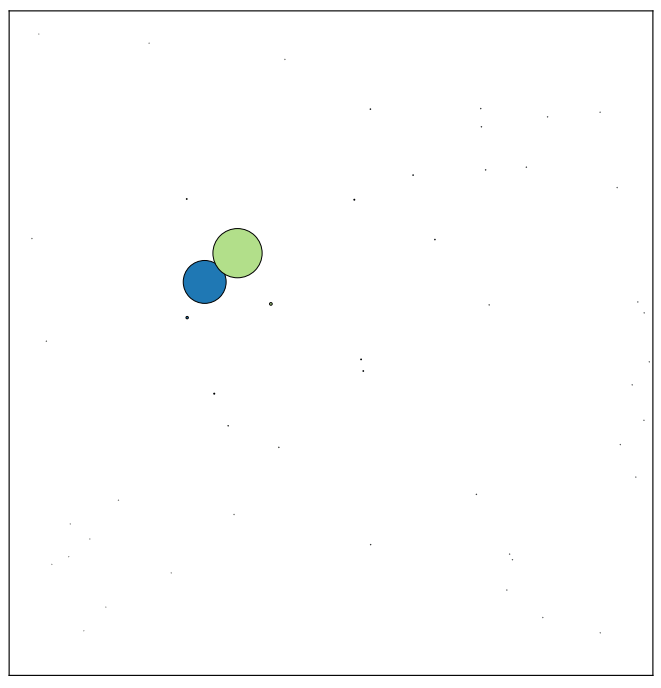

(a) $\phi=0.1$

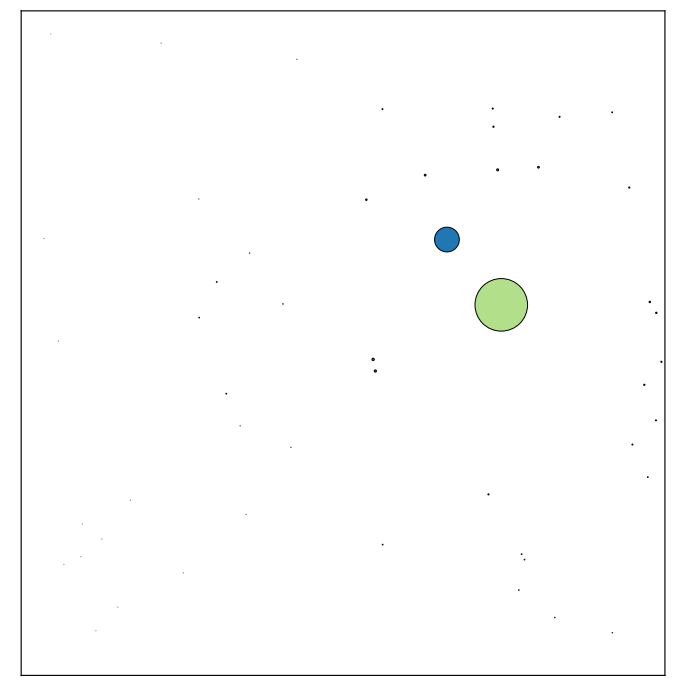

(b) $\phi=0.2$

Figure 5: Two different equilibria attained by the system when $a=0$ as $\phi_{1}$ is increased.

Our analysis in a lower dimensional setting in Section 3.2 identified the loss of stability of the trivial equilibrium as the support parameters are varied. This loss of stability is a bifurcation, which is likely to manifest as a sudden change in the resulting long-term behaviour of the system. For nonlinear systems, the behaviour of the system on one side of the bifurcation can be dramatically different from its behaviour on the other. That is, a small change in parameters can have a disproportionate effect on the resulting dynamics. We now determine whether a bifurcation can also be detected in the present high dimensional scenario.

In an effort to detect a bifurcation, we consider the case where $a=0$. Taking $\sigma_{1}=\sigma_{2}=1, \epsilon_{1}=\epsilon_{2}=0.1, b=1, \beta=3, \alpha=1, \rho_{1}=\rho_{2}=0.95$ and $\phi_{2}=0.01$, leads to $\tilde{\phi}_{1} \approx 0.11$, where $\tilde{\phi}_{1}$ is given in Theorem 1 . Taking uniform initial conditions, whereby the value of hostility is initially set to be equal to one at all locations for each side, we plot the resulting equilibrium as obtained via numerical simulation for two different values of $\phi_{1}$ in Figure $5^{1}$. In subfigure $5 \mathrm{a}, \phi_{1}=0.1$ and the conflict is overwhelmingly centered on

\footnotetext{
${ }^{1}$ Since $\rho_{1}$ and $\rho_{2}$ are large in comparison to the examples in Figure 4, the absolute size of the points in the figure have been rescaled. The size of the points across these two figures cannot be taken as a relative increase or decrease in hostility in comparison to
} 
two points towards the left of the unit square, in green territory. In subfigure $5 \mathrm{~b}, \phi_{1}=0.2$ and, in this case, the conflict is overwhelmingly centered on two points to the right of centre, this time in blue territory. This result echoes our finding from the lower dimensional setting that a bifurcation occurs as $\phi_{1}$ is increased through $\tilde{\phi}_{1}$ when $a=0$. A small change in the value of $\phi_{1}$ results in two different equilibria, in which the majority of the resulting hostility at equilibrium is found at two very different locations on $\mathcal{M}$. The value of the support parameter can therefore have a material effect on where the conflict is likely to escalate ${ }^{2}$.

We have demonstrated that features of the model which were identified analytically in low dimensional settings in Sections 3 can also be observed in a numerical example with a randomly generated spatial configuration. Scenarios with different parameters behave as expected when using this configuration. When the support mechanism is included in the model, we see different results from the model with no support. The dependence of the resulting equilibrium on the parameter $a$ has been explored, with the equilibrium for $a=0$ appearing more evenly distributed due to the support term. When $a=1$, we see concentration of hostility among those locations that are more accessible to the rest of their group. In addition, we present evidence that a bifurcation occurs as $\phi_{1}$ is increased in a location predicted by analysis of the model in low dimensional settings.

\section{Discussion}

In this paper, we have proposed a general spatial model of conflict escalation which describes the accumulation and allocation of resources at the locations occupied by adversaries engaged in hostile interaction. As well as 'arms race'-type growth effects, the model also incorporates an additional im-

\footnotetext{
those points in Figure 4.

${ }^{2}$ We also considered the case where $a=1$. Although our simulation was able to replicate the loss of stability of the trival equilibrium in the case where $N=M=2$, as analysed in Section 3.2, it did not detect a similar bifurcation in the same location in this higher dimensional scenario. There might be a number of reasons for this. Perhaps the existence of the bifurcation identified in Section 3.2 relied upon the dimension and distance metric specified in that section. Alternatively, perhaps the bifurcation point was merely dependent on the distance metric and this higher dimensional scenario has forced the point to move to a different location. This remains an interesting topic for further enquiry.
} 
portant aspect of real-world conflict scenarios, which is the ability of actors to coordinate activity across their various positions. By adding a mechanism by which adversaries can reallocate their resources in response to need, we have aligned the model more closely than existing approaches with the integrated behaviour commonly observed in real-world conflict situations.

Our mathematical analysis investigates some low-dimensional cases for the stability properties of the model. The sensitivity of the dynamics to the interpretation of resource reallocation is illustrated; depending on the parameterisation of this within-adversary action term, two scenarios are identified with opposite impact regarding the distribution of resources across various positions of an adversary. In each scenario we describe the dependence of stability conditions on the model parameters.

Our simulation results explore the behaviour of the model in a number of settings which correspond to stylised conflict scenarios. When support is allocated proportionately to existing levels of expenditure, we observe a 'rich-get-richer' effect, in which pronounced escalation occurs at a small number of locations, to an even greater extent than is observed in the absence of support. This leads to the formation of clusters of hostility in particular spatial regions, which can be interpreted as representing a high risk of conflict outbreak. This result suggests that agglomerative behaviour, in which adversaries prioritise centralised accumulation of arms, ultimately promotes the localised escalation of threat.

When support is distributed purely on the basis of distance, however, simulation results suggest that the distribution of threat becomes much more spatially homogeneous. This implies that such behaviour has a moderating effect, dampening the tendency to focus escalation on a small number of focal points. Whether this situation is preferable from the perspective of international security is open to question (and, indeed, likely to vary with circumstance), but it seems intuitive that catastrophic outbreaks are less likely to occur when tension is spread more evenly across locations.

A number of possible directions for further work can be identified in this area. From a mathematical perspective, a number of additional levels of complexity can be investigated for the model. Formal analysis for higherdimensional scenarios than those considered here, for example, would offer additional insight into these more realistic settings (and perhaps reveal further dynamic effects). Furthermore, an additional challenge concerns the treatment of uncertainty in the model. While our work here has assumed that all values are known, this is unlikely to be the case in the real-world: 
both parameters and resource levels may only be estimated. Establishing a means by which the robustness of the model to these issues can be investigated is a further challenge for work on this topic.

In terms of the model itself, there remains a need to calibrate the model against empirical data in order to understand which regimes are likely to apply in real-world scenarios. Furthermore, future directions for work may consider how this model can be refined (or re-interpreted) in such a way that the outbreak of conflict itself can be identified. The model, in its current form, describes escalation in tension and threat, but does not yet consider the ultimate consequence of this: the transition from threat to hostile engagement. To do this would require consideration of the decision processes taken by adversarial actors, and may mandate a different modelling approach.

There may also be scope to adapt the model developed here - or elements of it - for application in other contexts. While motivated here by the military case, the notions of threat and resource-sharing which the model concerns are general notions which could apply to other forms of conflict. Most immediately, given that the original Richardson model was an adaptation of an ecological model, the approach described here may have applications in ecology, in particular in relation to species competition. The addition of space in our model, together with the inclusion of within-species dynamics, may afford greater insight in such cases. Alternatively, there are a number of other human processes which may be suitable for this approach: non-military forms of conflict, such as economic competition, are an example of this.

In summary, the work presented here extends work on the spatial disaggregation of ecological models. We have introduced a general framework for such dynamical systems which incorporates both inter- and intra- adversary coupling across spatial locations. This can be applied to a number of conflict scenarios in which a notion of 'threat' is exerted by one entity over another: this may be military spending, as in the original Richardson model, or another form of antagonistic behaviour. Furthermore, it has potential application in other domains, including the ecological settings in which models of competition between species are also applied. Our analysis demonstrates that the model gives rise to complex dynamics, highlighting the significance of spatial considerations in models of this type. 


\section{Acknowledgments}

DK acknowledges the support of the Cimplex project, funded by the European Commission in the area "FET Proactive: Global Systems Science" (GSS) as a Research and Innovation Action under the H2020 Framework programme, Grant agreement number 641191.

\section{Appendix A. Supporting analysis}

\begin{tabular}{|ll|}
\hline \hline$P, Q$ & adversaries \\
$x_{1}, \ldots, x_{N}$ & locations of $P$ \\
$y_{1}, \ldots, y_{M}$ & locations of $Q$ \\
$p_{j}$ & hostility at location $x_{j}$ of adversary $P$ \\
$q_{l}$ & hostility at location $y_{l}$ of adversary $Q$ \\
$d$ & metric for the distance between locations \\
$\sigma_{1}, \sigma_{2}$ & decline rate of hostility of $P$ and $Q$ \\
$\rho_{1}, \rho_{2}$ & response rate of $P$ and $Q$ to the acquisition of the other \\
$\epsilon_{1}, \epsilon_{2}$ & external grievance of $P$ and $Q$ \\
$\phi_{1}, \phi_{2}$ & within-adversary support rate of $P$ and $Q$ \\
$b, \beta$ & parameters related to between-adversary actions \\
$a, \alpha$ & parameters related to within-adversary actions \\
\hline
\end{tabular}

Table A.1: Model variables and parameters.

\section{Aggregation of the model (3)}

We show now that our spatially-explicit model (3) is a disaggregation of the original Richardson model (1). Let $p:=\sum_{j=1}^{N} p_{j}$ and $q:=\sum_{l=1}^{M} q_{l}$ denote the total hostility of each adversary. First, we derive by the following computations that the total volume of resource redistributed amongst the locations of an adversary adds up to zero:

$$
\begin{gathered}
\sum_{j=1}^{N}\left(-\phi_{1} p_{j}+\phi_{1} \sum_{i=1}^{N} p_{i} \frac{p_{j}^{a} e^{-\alpha \cdot d\left(x_{j}, x_{i}\right)}}{\sum_{j^{\prime}=1}^{N} p_{j^{\prime}}^{a} e^{-\alpha \cdot d\left(x_{j^{\prime}}, x_{i}\right)}}\right) \\
=-\sum_{j=1}^{N} \phi_{1} p_{j}+\phi_{1} \sum_{i=1}^{N} p_{i} \frac{\sum_{j=1}^{N} p_{j}^{a} e^{-\alpha \cdot d\left(x_{j}, x_{i}\right)}}{\sum_{j^{\prime}=1}^{N} p_{j^{\prime}}^{a} e^{-\alpha \cdot d\left(x_{j^{\prime}}, x_{i}\right)}}=-\phi_{1} p+\phi_{1} p=0,
\end{gathered}
$$


with an equivalent relationship being satisfied for $q$. Second, the equalities

$$
\begin{aligned}
\sum_{j=1}^{N} \frac{\epsilon_{1}}{N} & =\epsilon_{1}, & \sum_{k=1}^{M} \frac{\epsilon_{2}}{M}=\epsilon_{2}, \\
-\sum_{j=1}^{N} \sigma_{1} p_{j} & =-\sigma_{1} p, & -\sum_{l=1}^{M} \sigma_{2} q_{l}=-\sigma_{2} q \\
\sum_{j=1}^{N} \rho_{1} \sum_{k=1}^{M} q_{k} \frac{p_{j}^{b} e^{-\beta \cdot d\left(x_{j}, y_{k}\right)}}{\sum_{i=1}^{N} p_{i}^{b} e^{-\beta \cdot d\left(x_{i}, y_{k}\right)}} & =\rho_{1} q, & \sum_{l=1}^{M} \rho_{2} \sum_{i=1}^{N} p_{i} \frac{q_{l}^{b} e^{-\beta \cdot d\left(x_{i}, y_{l}\right)}}{\sum_{k=1}^{M} q_{k}^{b} e^{-\beta \cdot d\left(x_{i}, y_{k}\right)}}=\rho_{2} p
\end{aligned}
$$

hold, thereby confirming that the aggregated volumes $p$ and $q$ are described by the original Richardson model (1).

\section{Global behaviour of system (4)}

The equilibrium equation reads

$$
A r^{2}+B r+C=0
$$

where, with $x=\frac{1+e^{-\alpha}+2 \phi_{1} e^{-\alpha}}{1+e^{-\alpha}} \bar{p}, y=\rho_{1} \bar{q}$

$$
\begin{aligned}
A & =\frac{1+e^{-\alpha}+2 \phi_{1} e^{-\alpha}}{1+e^{-\alpha}}\left(1-e^{-\beta\left(d_{2}-d_{1}\right)}\right)=\left(1-e^{-\beta\left(d_{2}-d_{1}\right)}\right) x / \bar{p}, \\
B & =\frac{1+e^{-\alpha}+2 \phi_{1} e^{-\alpha}}{1+e^{-\alpha}}\left(1+e^{-\beta\left(d_{2}-d_{1}\right)}\right) \bar{p}-\rho_{1} \bar{q}\left(1+e^{-\beta\left(d_{2}-d_{1}\right)}\right) \\
& =\left(1+e^{-\beta\left(d_{2}-d_{1}\right)}\right)(x-y), \\
C & =-\rho_{1} \bar{q}\left(1-e^{-\beta\left(d_{2}-d_{1}\right)}\right) \bar{p}=-\left(1-e^{-\beta\left(d_{2}-d_{1}\right)}\right) \bar{p} y .
\end{aligned}
$$

We can assume without loss of generality that $d_{2} \geq d_{1}$. Therefore we note that $A \geq 0, C \geq 0$, and $B>0$ by the observation that $x>y$ (which holds since $\left.\rho_{1} \rho_{2}<1\right)$.

If $d_{2}=d_{1}$, then the limiting equation reduces to

$$
\dot{r}=-r \frac{1+e^{-\alpha}+2 \phi_{1} e^{-\alpha}}{1+e^{-\alpha}}+\rho_{1} r \frac{\bar{q}}{\bar{p}} .
$$

Only one equilibrium $\bar{r}=0$ exists for this equation, and this is globally asymptotically stable. Indeed, stability follows from the condition $\frac{1+e^{-\alpha}+2 \phi_{1} e^{-\alpha}}{1+e^{-\alpha}}>$ 
$\rho_{1} \bar{q} / \bar{p}$, which holds since $\frac{1+e^{-\alpha}+2 \phi_{1} e^{-\alpha}}{1+e^{-\alpha}} \geq 1$ by $\phi \geq 0$ and $1>\rho_{1} \bar{q} / \bar{p}$ by $\rho_{1} \rho_{2}<1$.

If $d_{2}>d_{1}$ then $C / A<0$ holds, so there is a unique positive real root and a unique negative real root, given by

$$
\begin{aligned}
(\bar{r})_{ \pm} & =\frac{1}{2}\left(-B \pm \sqrt{B^{2}-4 A C}\right) \\
& =\frac{1}{2}(x-y)\left(-\left(1+e^{-\beta\left(d_{2}-d_{1}\right)}\right) \pm \sqrt{\left(\left(1+e^{-\beta\left(d_{2}-d_{1}\right)}\right)^{2}+4 e^{-\beta\left(d_{2}-d_{1}\right)}\right)}\right) .
\end{aligned}
$$

The right hand side of the limiting equation is valid for all $r$, it is zero if and only if $r=\bar{r}_{+}$or $r=\bar{r}_{-}$, and at $r=0$ the right hand side is clearly positive. This implies that $\dot{r}$ is positive for all $r$ such that $\bar{r}_{-}<r<\bar{r}_{+}$, and negative for all $r$ such that $r>\bar{r}_{+}$or $r<\bar{r}_{-}$. Also, the right hand side is positive at $r=-\bar{p}$ and negative at $r=\bar{p}$, so $\bar{r}_{-}<-\bar{p}$ and $\bar{r}_{+}>\bar{p}$ hold. The initial condition for the equations satisfies $\left|r_{0}\right| \leq \bar{p}$, and therefore $\bar{r}_{+}$is GAS.

\section{Stability analysis of system (6)}

First, we recall some definitions from [14]. We denote by $Z$ the set of all real square matrices whose off-diagonal entries are all non-positive. We say that a square matrix $A$ from the class $Z$ is a non-singular M-matrix if there exists a matrix $C \geq 0$ and a number $k>\rho(C)$ such that $A=k I-C$, where $I$ denotes the identity matrix of the same type as $A$, and $\rho(C)$ is the dominant eigenvalue of $C$. Theorem 5.1 in Ref. [14] establishes several alternative definitions for non-singular M-matrices: for instance, a matrix $A$ from the class $Z$ is a non-singular M-matrix if $A^{-1} \geq 0$. We denote by $s(A)$ the maximum real part of all eigenvalues of $A$, and say that a matrix is non-negative if all entries are non-negative. The following result is useful, and can be proved by similar arguments to those in Theorem 2 of [15].

Proposition 1. For a square matrix $J$, consider a splitting $J=F-V$ where $F$ is a non-negative matrix and $V$ is a non-singular M-matrix. Then it holds that $s(J)<0$ if and only if $\rho\left(F \cdot V^{-1}\right)<1, s(J)=0$ if and only if $\rho\left(F \cdot V^{-1}\right)=1$, and $s(J)>0$ if and only if $\rho\left(F \cdot V^{-1}\right)>1$.

Next we review some results from Refs. [16] and [17] that we are going to use several times in our proofs. Consider a nonnegative square matrix $K$, and consider another square matrix $K_{S}$ of the same type, defined using $K$ :

$$
\left[K_{S}\right]_{i j}=[K]_{i j} \text { or } 0 \quad \forall i, j .
$$


If $\rho\left(K-K_{S}\right)<1$ then it is meaningful to define

$$
\mathcal{T}=\rho\left(K_{S}\left(I-K+K_{S}\right)^{-1}\right)
$$

The following result is given in Theorem 2.1 of Ref. [16]: if $K$ is an irreducible matrix then $\mathcal{T}<1$ if and only if $\rho(K)<1, \mathcal{T}=1$ if and only if $\rho(K)=1$, and $\mathcal{T}>1$ if and only if $\rho(K)>1$.

In the theory of continuous dynamical systems it is well known that the stability of an equilibrium is determined by the eigenvalues of the Jacobian. More precisely, for a Jacobian $J$ the equilibrium is locally asymptotically stable if and only if all eigenvalues of $J$ have negative real part $(s(J)<0)$, and it is unstable if there is an eigenvalue with positive real part $(s(J)>0)$.

We recall that $J_{1}$ denotes the Jacobian of system (6). Let $V_{1}=\operatorname{diag}(1+$ $\left.\phi_{1}(1-\omega), 1+\phi_{2}(1-\omega)\right)$, which is a non-singular M-matrix, and let $F_{1}=J_{1}+$ $V_{1}$, which is non-negative. With these definitions we can obtain a splitting of the Jacobian and calculate $K_{1}=F_{1} \cdot V_{1}^{-1}$,

$$
K_{1}=\left(\begin{array}{cc}
\frac{\rho_{1}\left(1-\eta^{2}\right)}{1+\phi_{1}(1-\omega)} & \frac{\rho_{1} \eta}{1+\phi_{2}(1-\omega)} \\
\frac{\rho_{2} \eta}{1+\phi_{1}(1-\omega)} & \frac{\rho_{2}\left(1-\eta^{2}\right)}{1+\phi_{2}(1-\omega)}
\end{array}\right) .
$$

It follows by Proposition 1 that the trivial solution for the model is unstable if and only if $\rho\left(K_{1}\right)>1$, and that it is locally asymptotically stable if and only if $\rho\left(K_{1}\right)<1$. Moreover, $\rho\left(K_{1}\right)=1$ gives a threshold for stability. Since $\rho_{1} \rho_{2}<1$ by assumption, it is impossible that $\rho_{1}\left(1-\eta^{2}\right) \geq 1$ and $\rho_{2}\left(1-\eta^{2}\right) \geq 1$ hold simultaneously, and we therefore assume without loss of generality that $\rho_{1}\left(1-\eta^{2}\right)<1$. With the following result the proof of Theorem 1 is complete.

Proposition 2. If $\rho_{2}\left(1-\eta^{2}\right) \geq 1+\phi_{2}(1-\omega)$ then the trivial solution $(\bar{r}, \bar{s})=$ $(0,0)$ is unstable. If $\rho_{2}\left(1-\eta^{2}\right)<1+\phi_{2}(1-\omega)$ then it is possible to give stability conditions in terms of $\phi_{1}$.

Proof. We define

$$
L_{1}=\left(\begin{array}{cc}
0 & \frac{\rho_{1} \eta}{1+\phi_{2}(1-\omega)} \\
0 & \frac{\rho_{2}\left(1-\eta^{2}\right)}{1+\phi_{2}(1-\omega)}
\end{array}\right),
$$

and find that $\rho\left(L_{1}\right)=\left(\rho_{2}\left(1-\eta^{2}\right)\right) /\left(1+\phi_{2}(1-\omega)\right)$. For the non-negative matrices $K_{1}$ and $L_{1}$ it holds that $K_{1} \geq L_{1}$ (with the relation interpreted entry-wise), and it follows from Lemma 4.6 of Ref. [14] that $\rho\left(K_{1}\right) \geq \rho\left(L_{1}\right)$. 
Therefore if $\rho_{2}\left(1-\eta^{2}\right)>1+\phi_{2}(1-\omega)$ then $\rho\left(K_{1}\right)>1$ holds, and $\rho\left(K_{1}\right) \geq 1$ holds if $\rho_{2}\left(1-\eta^{2}\right)=1+\phi_{2}(1-\omega)$. In the latter case, however, it is easy to check that 1 is not an eigenvalue of $K_{1}$, and therefore $\rho\left(K_{1}\right)>1$ must hold.

If $\rho_{2}\left(1-\eta^{2}\right)<1+\phi_{2}(1-\omega)$ then $\rho\left(L_{1}\right)<1$ so it is meaningful to define

$$
\left(I_{2}-L_{1}\right)^{-1}=\left(\begin{array}{cc}
1 & \frac{\rho_{1} \eta}{1+\phi_{2}(1-\omega)-\rho_{2}\left(1-\eta^{2}\right)} \\
0 & \frac{1+\phi_{2}(1-\omega)}{1+\phi_{2}(1-\omega)-\rho_{2}\left(1-\eta^{2}\right)}
\end{array}\right)
$$

and we calculate $\mathcal{T}_{1}:=\rho\left(\left(K_{1}-L_{1}\right)\left(I_{2}-L_{1}\right)^{-1}\right)$, as

$$
\mathcal{T}_{1}=\frac{1}{1+\phi_{1}(1-\omega)}\left(\rho_{1}\left(1-\eta^{2}\right)+\frac{\rho_{1} \eta \rho_{2} \eta}{1+\phi_{2}(1-\omega)-\rho_{2}\left(1-\eta^{2}\right)}\right) .
$$

By the irreducibility of $K_{1}$ it holds by Theorem 2.1 of Ref. [16] that $\rho\left(K_{1}\right)>1$ if and only if $\mathcal{T}_{1}>1, \rho\left(K_{1}\right)=1$ if and only if $\mathcal{T}_{1}=1$, and $\rho\left(K_{1}\right)<1$ if and only if $\mathcal{T}_{1}<1$. In particular, the surface in the parameter space that separates the regions of stability and instability is described by

$$
\mathcal{T}_{1}=1 \quad \Leftrightarrow \quad \rho_{1} \rho_{2} \eta^{2}=\left(1+\phi_{1}(1-\omega)-\rho_{1}\left(1-\eta^{2}\right)\right)\left(1+\phi_{2}(1-\omega)-\rho_{2}\left(1-\eta^{2}\right)\right)
$$

and a stability threshold for $\phi_{1}$ is given as

$$
\tilde{\phi}_{1}:=\left(\rho_{1}\left(1-\eta^{2}\right)+\frac{\rho_{1} \eta \rho_{2} \eta}{1+\phi_{2}(1-\omega)-\rho_{2}\left(1-\eta^{2}\right)}-1\right) /(1-\omega),
$$

that is, the trivial solution is locally asymptotically stable $\left(\mathcal{T}_{1}<1\right)$ if and only if $\phi_{1}>\tilde{\phi}_{1}$. Note that depending on other parameters $\tilde{\phi}_{1}$ might be negative in which case stability holds for all values of $\phi_{1}$ in the feasible parameter space, however it is possible in any case to stabilise the trivial solution by increasing $\phi_{1}$ through $\tilde{\phi}_{1}$.

\section{Stability analysis of system (7)}

Let $F_{2}=J_{2}+I_{2}$ and $V_{2}=I_{2}$, where $I_{2}$ denotes the identity matrix of order 2. By $\phi_{1} \omega(1-\omega)>0$ and $\phi_{2} \omega(1-\omega)>0$, we observe that $F_{2}$ is a non-negative matrix and $V_{2}$ in a non-singular M-matrix. Furthermore, these definitions allow $J_{2}$ to be decomposed as $F_{2}-V_{2}$. Defining $K_{2}=F_{2} \cdot V_{2}^{-1}$, it follows by Proposition 1 that the trivial equilibrium $(\bar{r}, \bar{s})=(0,0)$ is locally asymptotically stable if $\rho\left(K_{2}\right)<1$, whereas it is unstable if $\rho\left(K_{2}\right)>1$.

$$
K_{2}=\left(\begin{array}{cc}
\phi_{1} \omega(1-\omega)+\rho_{1}\left(1-\eta^{2}\right) & \rho_{1} \eta \\
\rho_{2} \eta & \phi_{2} \omega(1-\omega)+\rho_{2}\left(1-\eta^{2}\right)
\end{array}\right)
$$


Proposition 3. If either $\phi_{1} \omega(1-\omega)+\rho_{1}\left(1-\eta^{2}\right) \geq 1$ or $\phi_{2} \omega(1-\omega)+\rho_{2}(1-$ $\left.\eta^{2}\right) \geq 1$ holds then the trivial equilibrium $(\bar{r}, \bar{s})=(0,0)$ is unstable.

Proof. Assume that $\phi_{2} \omega(1-\omega)+\rho_{2}\left(1-\eta^{2}\right) \geq 1$, and consider the matrix

$$
L_{2}=\left(\begin{array}{cc}
0 & 0 \\
\rho_{2} \eta & \phi_{2} \omega(1-\omega)+\rho_{2}\left(1-\eta^{2}\right)
\end{array}\right)
$$

It holds that $L_{2}$ is a nonnegative matrix and $L_{2} \leq K_{2}$, which implies by Lemma 4.6 of Ref. [14] that $\rho\left(L_{2}\right) \leq \rho\left(K_{2}\right)$. Observing that $\rho\left(L_{2}\right)=\phi_{2} \omega(1-$ $\omega)+\rho_{2}\left(1-\eta^{2}\right) \geq 1$ we conclude that $\rho\left(K_{2}\right) \geq 1$. Equality can hold if and only if $\phi_{2} \omega(1-\omega)+\rho_{2}\left(1-\eta^{2}\right)=1$, but in this case 1 is not an eigenvalue of $K_{2}$ and therefore $\rho\left(K_{2}\right)>1$ must hold. A similar conclusion is obtained when $\phi_{1} \omega(1-\omega)+\rho_{1}\left(1-\eta^{2}\right) \geq 1$ holds. The proof is complete.

Following the last result, we note that if $\phi_{2} \omega(1-\omega)+\rho_{2}\left(1-\eta^{2}\right) \geq 1$ then the trivial equilibrium $(\bar{r}, \bar{s})=(0,0)$ is unstable for all values of $\phi_{1}$ and $\rho_{1}$. In particular, if $\phi_{2} \omega(1-\omega) \geq 1$ then the trivial equilibrium $(\bar{r}, \bar{s})=(0,0)$ is unstable for all values of $\phi_{1}, \rho_{1}$ and $\beta$.

The case when $\phi_{2} \omega(1-\omega)+\rho_{2}\left(1-\eta^{2}\right)<1$ is not necessary unstable, however. Next we establish conditions under which the within-adversary interaction term, via $\phi_{1}$, is capable of stabilising the equilibrium in this case. We use the fact that $\rho\left(K_{2}\right)=1$ establishes a boundary for stability. The next result completes the proof of Theorem 2 .

Proposition 4. If $\phi_{2} \omega(1-\omega)+\rho_{2}\left(1-\eta^{2}\right)<1$ then it is possible to give stability conditions in terms of $\phi_{1}$.

Proof. Assume that $\phi_{2} \omega(1-\omega)+\rho_{2}\left(1-\eta^{2}\right)<1$. Then by $\rho\left(L_{2}\right)<1$ the number

$$
\mathcal{T}_{2}=\rho\left(\left(K_{2}-L_{2}\right) \cdot\left(I_{2}-L_{2}\right)^{-1}\right)
$$

is well-defined. Straightforward computations yield

$$
\mathcal{T}_{2}=\phi_{1} \omega(1-\omega)+\rho_{1}\left(1-\eta^{2}\right)+\frac{\rho_{1} \rho_{2} \eta^{2}}{1-\phi_{2} \omega(1-\omega)-\rho_{2}\left(1-\eta^{2}\right)},
$$

and we have by Ref. [16] that $\rho\left(K_{2}\right)>1$ if and only if $\mathcal{T}_{2}>1, \rho\left(K_{2}\right)=1$ if and only if $\mathcal{T}_{2}=1$, and $\rho\left(K_{2}\right)<1$ if and only if $\mathcal{T}_{2}<1$. In particular, the equation $\mathcal{T}_{2}=1$ defines the stability boundary

$$
\left(\phi_{1} \omega(1-\omega)+\rho_{1}\left(1-\eta^{2}\right)-1\right)\left(\phi_{2} \omega(1-\omega)+\rho_{2}\left(1-\eta^{2}\right)-1\right)=\rho_{1} \rho_{2} \eta^{2} .
$$


In particular, if $\phi_{1}=\phi_{2}=0$ then the curve separating the stable region from the unstable region in the $\left(\rho_{1}, \rho_{2}\right)$-plane is given by $\rho_{2}=\frac{\rho_{1}\left(1-\eta^{2}\right)-1}{\left(\rho_{1}\left(1-\eta^{2}\right)-1\right)\left(1-\eta^{2}\right)-\rho_{1} \eta^{2}}$. A stability threshold for $\phi_{1}$ arises from the condition $\mathcal{T}_{2}=1$; specifically,

$$
\breve{\phi}_{1}=\left(1-\rho_{1}\left(1-\eta^{2}\right)-\frac{\rho_{1} \rho_{2} \eta^{2}}{1-\phi_{2} \omega(1-\omega)-\rho_{2}\left(1-\eta^{2}\right)}\right) /(\omega(1-\omega)),
$$

and the trivial solution is locally asymptotically stable if and only if $\phi_{1}<\breve{\phi}_{1}$. Note that $\breve{\phi}_{1}$ might be negative, in which case the trivial solution is unstable for all $\phi_{1}$.

For the proof of Theorem 3, we define

$$
L_{3}=\left(\begin{array}{cc}
\phi_{1} \omega(1-\omega)+\rho_{1}\left(1-\eta^{2}\right) & 0 \\
0 & \phi_{2} \omega(1-\omega)+\rho_{2}\left(1-\eta^{2}\right)
\end{array}\right)
$$

and note by $\rho\left(K_{2}-L_{3}\right)=\sqrt{\rho_{1} \rho_{2} \eta^{2}}<1$ that the matrix $I_{2}-K_{2}+L_{3}$ is invertible, so that it is meaningful to define

$$
\mathcal{T}_{3}=\rho\left(L_{3} \cdot\left(I_{2}-K_{2}+L_{3}\right)^{-1}\right) .
$$

Unlike in the analysis for $\mathcal{T}_{1}$ and $\mathcal{T}_{2}$, no closed form expression can be derived for $\mathcal{T}_{3}$; however its value can be computed numerically.

Proof of Theorem ??. By Theorem 2.1 of Ref. [16, 17], we have that $\rho\left(K_{2}\right)>1$ if and only if $\mathcal{T}_{3}>1$ (instability), $\rho\left(K_{2}\right)=1$ if and only if $\mathcal{T}_{3}=1$ (stability boundary), and $\rho\left(K_{2}\right)<1$ if and only if $\mathcal{T}_{3}<1$ (local asymptotic stability).

We define by $K_{2}^{*}$ the matrix obtained from $K_{2}$ by replacing $\left[K_{2}\right]_{1,1}$ by $\left[K_{2}\right]_{1,1} / \mathcal{T}_{3}$ and $\left[K_{2}\right]_{2,2}$ by $\left[K_{2}\right]_{2,2} / \mathcal{T}_{3}$. By Theorem 2.2 of Ref. [16], $\rho\left(K_{2}^{*}\right)=$ 1. We observe that replacing $\phi_{1}$ by $\phi_{1}^{*}$ in $K_{2}$ makes $\left[K_{2}\right]_{1,1}$ transform into $\left[K_{2}\right]_{1,1} / \mathcal{T}_{3}=\left[K_{2}^{*}\right]_{1,1} ;$ indeed,

$$
\left(\frac{\phi_{1}}{\mathcal{T}_{3}}+\frac{\rho_{1}\left(1-\eta^{2}\right)}{\omega(1-\omega)}\left(\frac{1}{\mathcal{T}_{3}}-1\right)\right) \omega(1-\omega)+\rho_{1}\left(1-\eta^{2}\right)=\frac{\phi_{1} \omega(1-\omega)+\rho_{1}\left(1-\eta^{2}\right)}{\mathcal{T}_{3}} .
$$

It can be shown by similar computations that changing $\phi_{2}$ to $\phi_{2}^{*}$ results in $\left[K_{2}\right]_{2,2}$ changing to $\left[K_{2}^{*}\right]_{2,2}$, and therefore we conclude that such transformations bring the matrix $K_{2}$ to $K_{2}^{*}$. It is easy to see that $\phi_{1}<\phi_{1}^{*}$ and $\phi_{2}<\phi_{2}^{*}$ if $\mathcal{T}_{3}<1$, and the inequalities are reversed if $\mathcal{T}_{3}>1$. Therefore if $\mathcal{T}_{3}<1$ 
(equivalently, $\rho\left(K_{2}\right)<1$ ), then the transformations $\phi_{1} \rightarrow \phi_{1}^{*}$ and $\phi_{2} \rightarrow \phi_{2}^{*}$ increase the support parameters, and this leads to destabilisation since $K_{2}$ transforms into $K_{2}^{*}$ and $\rho\left(K_{2}^{*}\right)=1$. At the same time, if $\mathcal{T}_{3}>1$ (that is, the trivial solution is unstable by $\rho\left(K_{2}\right)>1$ ), then the transformations decrease the support parameters and the spectral radius, leading to stabilisation when $\rho\left(K_{2}\right)$ reaches 1 at $K_{2}=K_{2}^{*}$. However, $\phi_{1}^{*} \geq 0$ and $\phi_{2}^{*} \geq 0$ must hold in order for the transformations to be feasible, otherwise stabilisation remains impossible by controlling $\phi_{1}$ and $\phi_{2}$ only. It is easy to see that nonnegativity of $\phi_{1}^{*}$ and $\phi_{2}^{*}$ is equivalent to $\mathcal{T}_{3} \leq 1+\phi_{1} \omega(1-\omega) /\left(\rho_{1}\left(1-\eta^{2}\right)\right)$ and $\mathcal{T}_{3} \leq 1+\phi_{2} \omega(1-\omega) /\left(\rho_{2}\left(1-\eta^{2}\right)\right)$, respectively, and the proof is complete.

[1] F. W. Lanchester, Aircraft in Warfare: The Dawn of the Fourth Arm, Constable and Company, London, UK, 1916.

[2] L. F. Richardson, Arms and Insecurity, The Boxwood Press, Pittsburgh, PA, 1960.

[3] M. P. Atkinson, A. Gutfraind, M. Kress, When do armed revolts succeed: lessons from Lanchester theory, Journal of the Operational Research Society 63 (10) (2012) 1363-1373. doi:10.1057/jors.2011.146.

[4] L. Blank, C. E. Enomoto, D. Gegax, T. McGuckin, C. Simmons, A Dynamic Model of Insurgency: The Case of the War in Iraq, Peace Economics, Peace Science and Public Policy 14 (2). doi:10.2202/15548597.1120.

[5] M. D. Intriligator, D. L. Brito, A predator-prey model of guerrilla warfare, Synthese 76 (2) (1988) 235-244. doi:10.1007/BF00869590.

[6] S. Jackson, B. Russett, D. Snidal, D. Sylvan, Conflict and Coercion in Dependent States, Journal of Conflict Resolution 22 (4) (1978) 627-657. doi:10.1177/002200277802200404.

[7] M. Karmeshu, V. P. Jain, A. K. Mahajan, A Dynamic Model of Domestic Political Conflict Process, Journal of Conflict Resolution 34 (2) (1990) 252-269. doi:10.1177/0022002790034002004.

[8] M. Kress, N. J. MacKay, Bits or shots in combat? The generalized Deitchman model of guerrilla warfare, Operations Research Letters 42 (1) (2014) 102-108. doi:10.1016/j.orl.2013.08.004. 
[9] L. S. Liebovitch, V. Naudot, R. Vallacher, A. Nowak, L. Bui-Wrzosinska, P. Coleman, Dynamics of two-actor cooperation-competition conflict models, Physica A: Statistical Mechanics and its Applications 387 (25) (2008) 6360-6378. doi:10.1016/j.physa.2008.07.020.

[10] M. R. Qubbaj, R. Muneepeerakul, Two-actor conflict with time delay: A dynamical model, Physical Review E 86 (5) (2012) 056101. doi:10.1103/PhysRevE.86.056101.

[11] A. Rojas-Pacheco, B. Obregón-Quintana, L. S. Liebovitch, L. GuzmánVargas, Time-delay effects on dynamics of a two-actor conflict model, Physica A: Statistical Mechanics and its Applications 392 (3) (2013) 458-467. doi:10.1016/j.physa.2012.09.021.

[12] A. M. Saperstein, Chaos in models of arms races and the initiation of war: Crisis stability and instability in an international system, Complexity 12 (3) (2007) 22-26. doi:10.1002/cplx.20162.

[13] P. Baudains, H. Fry, T. Davies, A. Wilson, S. Bishop, A dynamic spatial model of conflict escalation, European Journal of Applied Mathematics 27 (Special Issue 03) (2016) 530-553. doi:10.1017/S0956792515000558.

[14] M. Fiedler, Special Matrices and Their Applications in Numerical Mathematics, Martinus Nijhoff, 1986.

[15] P. van den Driessche, J. Watmough, Reproduction numbers and sub-threshold endemic equilibria for compartmental models of disease transmission, Mathematical Biosciences 180 (1-2) (2002) 29-48. doi:10.1016/S0025-5564(02)00108-6.

[16] Z. Shuai, J. a. P. Heesterbeek, P. v. d. Driessche, Extending the type reproduction number to infectious disease control targeting contacts between types, Journal of Mathematical Biology 67 (5) (2012) 1067-1082. doi:10.1007/s00285-012-0579-9.

[17] Z. Shuai, J. a. P. Heesterbeek, P. v. d. Driessche, Erratum to: Extending the type reproduction number to infectious disease control targeting contacts between types, Journal of Mathematical Biology 71 (1) (2015) 255-257. doi:10.1007/s00285-015-0858-3. 